$12-1-2020$

\title{
Sleep Induction by Mechanosensory Stimulation in Drosophila.
}

\author{
Arzu Öztürk-Çolak \\ Thomas Jefferson University \\ Sho Inami \\ Thomas Jefferson University \\ Joseph R. Buchler \\ Thomas Jefferson University \\ Patrick D. McClanahan \\ University of Pennsylvania \\ Andri Cruz \\ Thomas Jefferson University
}

Follow this and additional works at: https://jdc.jefferson.edu/department_neuroscience See next page for additional authors

Part of the Neurosciences Commons

Let us know how access to this document benefits you

\section{Recommended Citation}

Öztürk-Çolak, Arzu; Inami, Sho; Buchler, Joseph R.; McClanahan, Patrick D.; Cruz, Andri; FangYen, Christopher; and Koh, Kyunghee, "Sleep Induction by Mechanosensory Stimulation in Drosophila." (2020). Department of Neuroscience Faculty Papers. Paper 52.

https://jdc.jefferson.edu/department_neuroscience/52

This Article is brought to you for free and open access by the Jefferson Digital Commons. The Jefferson Digital Commons is a service of Thomas Jefferson University's Center for Teaching and Learning (CTL). The Commons is a showcase for Jefferson books and journals, peer-reviewed scholarly publications, unique historical collections from the University archives, and teaching tools. The Jefferson Digital Commons allows researchers and interested readers anywhere in the world to learn about and keep up to date with Jefferson scholarship. This article has been accepted for inclusion in Department of Neuroscience Faculty Papers by an authorized administrator of the Jefferson Digital Commons. For more information, please contact: JeffersonDigitalCommons@jefferson.edu. 
Authors

Arzu Öztürk-Çolak, Sho Inami, Joseph R. Buchler, Patrick D. McClanahan, Andri Cruz, Christopher FangYen, and Kyunghee Koh 


\section{Cell Reports}

\section{Sleep Induction by Mechanosensory Stimulation in Drosophila}

\section{Graphical Abstract}

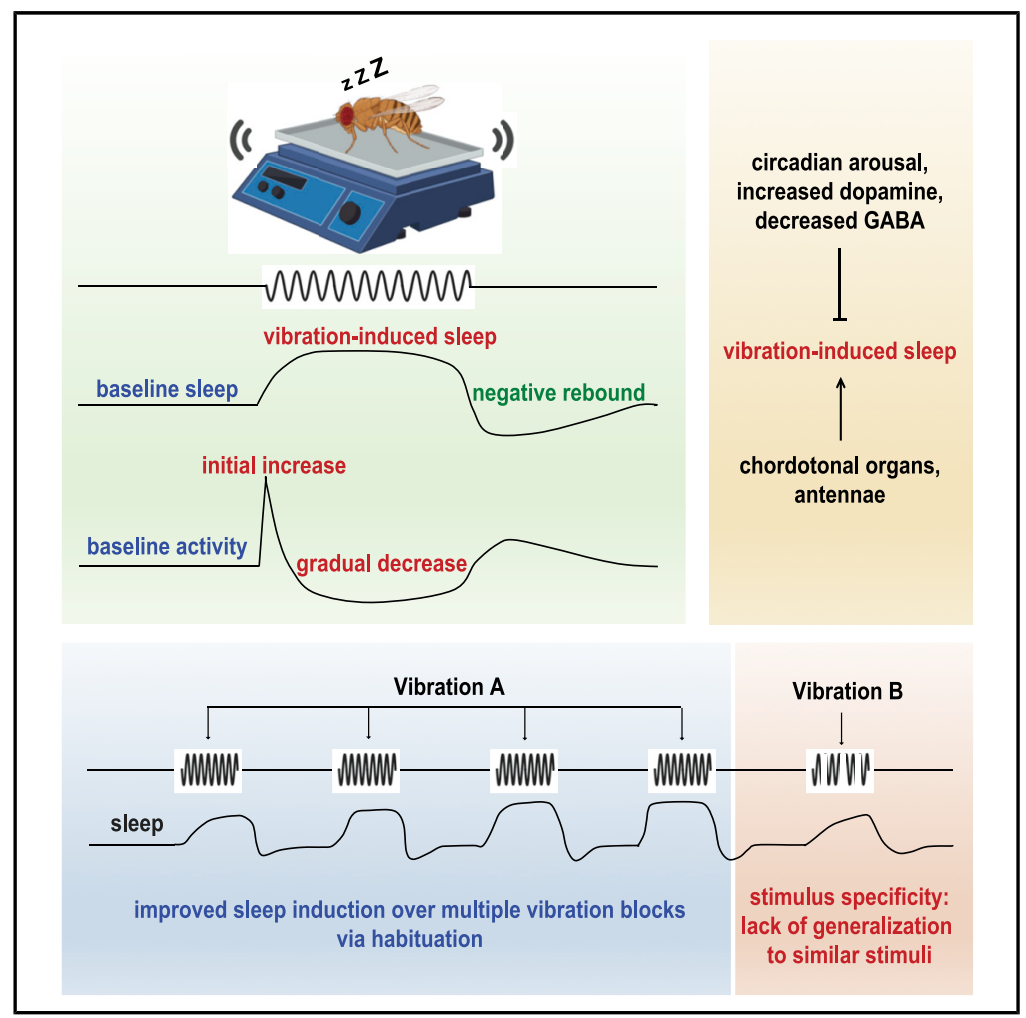

\section{Highlights}

- A variety of mechanical stimuli promote vibration-induced sleep (VIS) in Drosophila

- VIS leads to homeostatic sleep credit and reduced arousability

- Heightened arousal or reduced GABA signaling can suppress VIS

- Habituation learning improves sleep induction in successive blocks of vibration

\section{Authors}

Arzu Öztürk-Çolak, Sho Inami, Joseph R. Buchler, Patrick D. McClanahan, Andri Cruz, Christopher Fang-Yen, Kyunghee Koh

\section{Correspondence}

kyunghee.koh@jefferson.edu

\section{In Brief}

Öztürk-Çolak et al. demonstrate that gentle vibration induces sleep in Drosophila. The authors show that sleep induction improves over multiple vibration sessions, which suggests that habituation, a form of simple learning, plays a significant role in vibrationinduced sleep. 


\title{
Article \\ Sleep Induction by Mechanosensory Stimulation in Drosophila
}

\author{
Arzu Öztürk-Çolak, ${ }^{1,3}$ Sho Inami, ${ }^{1,3}$ Joseph R. Buchler, ${ }^{1}$ Patrick D. McClanahan, ${ }^{2}$ Andri Cruz, ${ }^{1}$ Christopher Fang-Yen, ${ }^{2}$ \\ and Kyunghee Koh ${ }^{1,4, *}$ \\ ${ }^{1}$ Department of Neuroscience, Jefferson Center for Synaptic Biology, and the Farber Institute for Neurosciences, Thomas Jefferson \\ University, Philadelphia, PA 19106, USA \\ ${ }^{2}$ Department of Bioengineering, University of Pennsylvania, Philadelphia, PA 19104, USA \\ ${ }^{3}$ These authors contributed equally \\ ${ }^{4}$ Lead Contact \\ ${ }^{*}$ Correspondence: kyunghee.koh@jefferson.edu \\ https://doi.org/10.1016/j.celrep.2020.108462
}

\section{SUMMARY}

People tend to fall asleep when gently rocked or vibrated. Experimental studies have shown that rocking promotes sleep in humans and mice. However, the mechanisms underlying the phenomenon are not well understood. A habituation model proposes that habituation, a form of non-associative learning, mediates sleep induction by monotonous stimulation. Here, we show that gentle vibration promotes sleep in Drosophila in part through habituation. Vibration-induced sleep (VIS) leads to increased homeostatic sleep credit and reduced arousability, and can be suppressed by heightened arousal or reduced GABA signaling. Multiple mechanosensory organs mediate VIS, and the magnitude of VIS depends on vibration frequency and genetic background. Sleep induction improves over successive blocks of vibration. Furthermore, training with continuous vibration does not generalize to intermittent vibration, demonstrating stimulus specificity, a characteristic of habituation. Our findings suggest that habituation plays a significant role in sleep induction by vibration.

\section{INTRODUCTION}

Anecdotal observations suggest that babies sleep better when gently rocked or bounced, and people tend to fall asleep during long car rides. Several experimental studies have confirmed that rocking promotes sleep in infants, adult humans, and mice (Bayer et al., 2011; Kompotis et al., 2019; Korner et al., 1978; Perrault et al., 2019). However, the underlying mechanisms are not well understood.

A model of how sensory stimulation promotes sleep, which we refer to as the habituation model, proposes that habituation, a form of non-associative learning, plays a critical role in sleep induction by monotonous stimulation (Pavlov, 1927; Sokolov, 1963; Bohlin, 1971). The model suggests that habituation to repeated stimuli reduces arousal and increases the propensity for sleep through a common mechanism. Habituation is traditionally viewed as a process that allows organisms to ignore predictable, unimportant stimuli so they can focus on salient changes in the environment (Rankin et al., 2009; Thompson and Spencer, 1966). According to this view, once an organism had learned to ignore monotonous stimuli, they would no longer be effective at inducing sleep. However, recent findings suggest that habituation is more than merely learning to ignore unimportant stimuli and allows organisms to switch between alternative behaviors, depending on environmental conditions (McDiarmid et al., 2019). Incorporating the more recent view of habituation, the model proposes that habituation allows organisms to choose sleep over wakefulness under monotonous stimulation conditions.
Habituation is a reduction in behavioral response that does not involve sensory adaptation or motor fatigue (Rankin et al., 2009). One way to distinguish habituation from sensory adaptation and motor fatigue is by demonstrating stimulus specificity, which refers to the phenomenon that habituation does not generalize to a novel stimulus within the same sensory modality as the habituated stimulus. Through stimulus specificity, organisms can reduce the response to repetitive insignificant stimuli while maintaining the ability to respond to novel, potentially significant stimuli.

Mechanosensory stimuli are processed by the auditory, vestibular (gravity sensing), somatosensory, and proprioceptive systems (Delmas et al., 2011; Tuthill and Wilson, 2016). The mammalian ear processes sound (vibration) and gravity in parallel auditory and vestibular systems, respectively (Ekdale, 2016). In the fly, the chordotonal organs in the antennae, wing bases, and legs constitute major mechanosensory systems that mediate audition, gravity and wind sensing, and proprioception (Albert and Göpfert, 2015; Tuthill and Wilson, 2016). A study in mice reported that rocking promotes sleep through the vestibular otolithic organs (Kompotis et al., 2019). However, several studies have demonstrated that repetitive acoustic stimuli can also enhance sleep slow waves in humans, presumably through the auditory system (Tononi et al., 2010; Bohlin, 1971). Together, these results suggest that mechanosensory stimuli can influence sleep through multiple sensory systems in mammals.

Here, we report that mechanosensory stimuli promote sleep in flies. Flies exhibited reduced sleep after vibration ("negative 


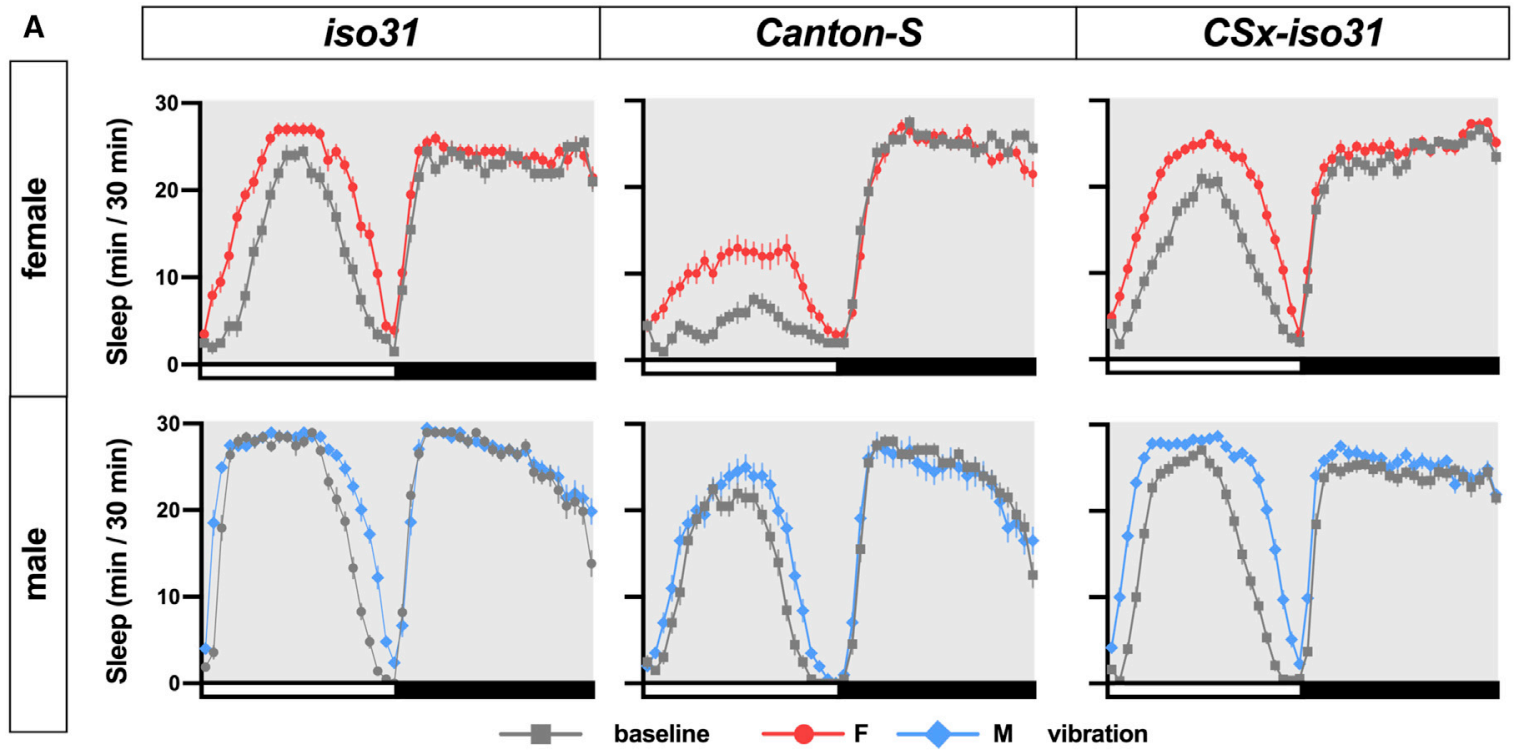

B
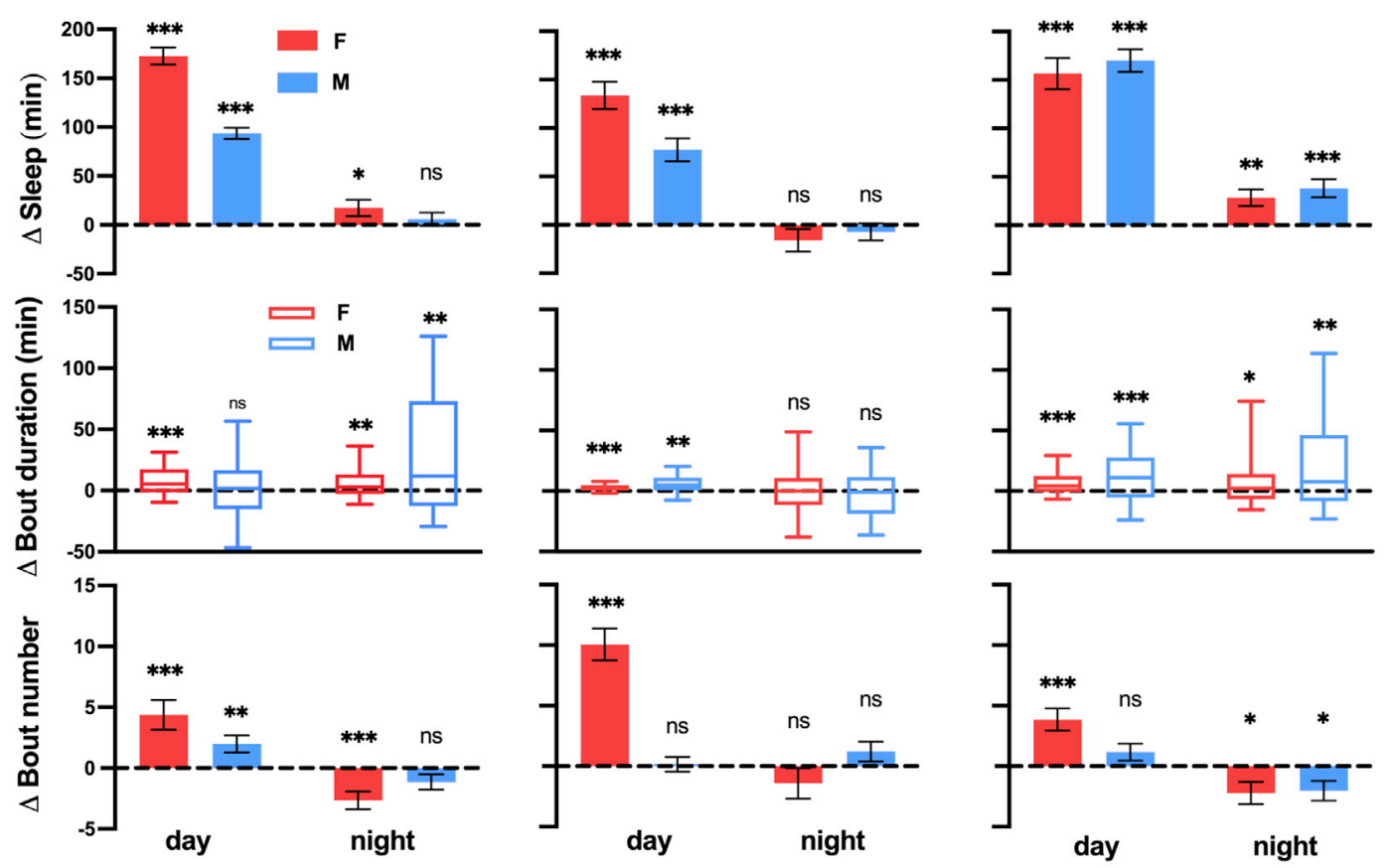

C

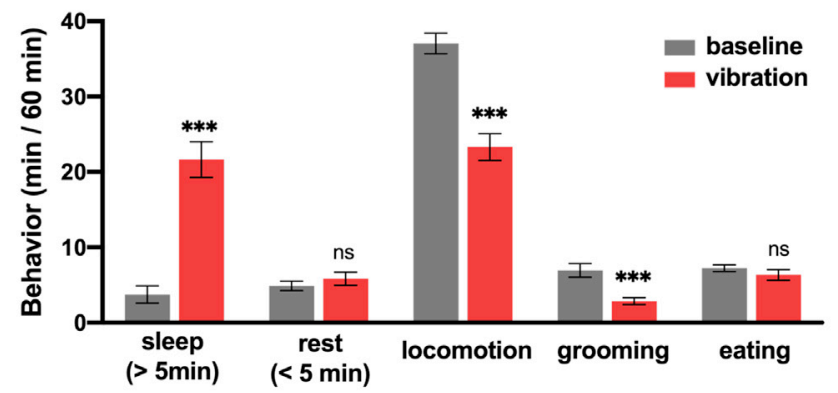


rebound"), which suggests vibration-induced sleep (VIS) leads to the accumulation of sleep credit. Flies exhibited reduced arousability during VIS relative to baseline sleep, which suggests that sleep during vibration is deeper than baseline sleep. Heightened arousal through the circadian clock, elevated dopamine signaling, and reduced GABA signaling suppressed VIS. More important, sleep induction improved over successive blocks of vibration, and VIS exhibited stimulus specificity, suggesting that habituation contributes to VIS. Ablation of the antennae or chordotonal organs partially suppressed VIS but did not eliminate it, indicating that multiple sensory organs are involved. By presenting simple sinusoidal vibrations to three control strains, we found that vibrations ranging from 3 to $200 \mathrm{~Hz}$ can induce sleep to varying degrees, depending on the frequency and genetic background. Our data suggest that habituation plays a critical role in sleep induction by mechanosensory stimulation.

\section{RESULTS}

\section{Gentle Mechanical Stimulation Promotes Sleep}

To test whether gentle mechanical stimuli can promote sleep in Drosophila, we placed Drosophila activity monitors (DAMs) on a shelf $\sim 40 \mathrm{~cm}$ above a multi-tube vortexer, such that a small amplitude vibration from the vortexer was coupled to the DAMs (Figure S1). After establishing a day of baseline sleep/ wake behavior, we applied continuous vibration for 1 day to 3 control strains in a 12-h light:12-h dark (LD) condition. iso31 (an isogenic white ${ }^{1,118}$ control strain commonly used for sleep research; Ryder et al., 2004), Canton-S (CS, a wild-type strain widely used in Drosophila research), and CSx-iso31 (a derivative of iso31, in which the X chromosome containing the white gene is replaced by that of the CS strain) were used to examine the effects of the genetic background on VIS. We found that daytime sleep in both males and females of all three strains was markedly increased during vibration (Figures $1 \mathrm{~A}$ and 1B). Nighttime VIS was modest or absent, suggesting that the circadian clock or light can modulate it.

To examine the changes in sleep architecture during vibration, we examined sleep bout duration and bout number. The substantial increase in daytime sleep during vibration in all three strains was due to increased bout duration and/or number (Figure 1B). In contrast, the modest nighttime sleep gain in iso31 females and CSx-iso31 flies was due to a combination of an increase in sleep bout duration and a decrease in sleep bout number (Figure 1B), suggesting that sleep was more consolidated during vibration. Vibration may promote sleep by influencing sleep initiation (reflected in increased sleep bout num- ber), maintenance (reflected in increased sleep bout duration), and consolidation (reflected in increased sleep bout duration and decreased bout number).

Notably, sleep during vibration exhibited a normal decrease toward the end of the light period (Figure 1A), demonstrating that the circadian arousal signal modulates VIS and that the flies did not have difficulty moving during vibration. Video recording of their behavior revealed that flies initially responded to vibration with increased locomotor activity (Video S1), further confirming that vibration did not cause paralysis or difficulty in locomotion. Video recording also showed that flies gradually became inactive during vibration and that they did not increase activities involving small movements such as eating or grooming that are not detected by the single-beam DAM system. Manual scoring of videos confirmed that vibration increased sleep time while decreasing time engaged in locomotion and grooming in iso31 females (Figure 1C). Vibration did not significantly affect the time for eating or brief $(<5 \mathrm{~min})$ rest.

For a high-throughput sleep analysis that takes local movements into account, we used multi-beam monitors containing 17 infrared beams. Multi-beam monitors allowed the measurement of local (intra-beam) movements that occur within a single beam such as grooming, as well as beam-to-beam (inter-beam) movements. As previously shown (Garbe et al., 2015), sleep measured using multi-beam monitors was markedly lower than that measured using single-beam monitors (compare Figures 1 and 2). More important, we observed a substantial daytime sleep gain during vibration in LD using multi-beam monitors, even when local movements were included (Figures $2 \mathrm{~A}$ and $2 \mathrm{~B}$ ). Multi-beam data also confirmed that nighttime VIS was modest or absent. An examination of activity counts (combined intraand inter-beam counts) showed that flies initially showed increased activity in response to vibration, but their activity decreased gradually to levels below the baseline level (Figures $2 \mathrm{C}$ and $2 \mathrm{D}$ ). A previous study also observed a sharp initial increase, followed by a gradual decline in activity during the first hour of vibration (Simoni et al., 2014). Our results further showed that activity declined below the baseline as vibration continued. The multi-beam data validated the conclusion based on the single-beam data that vibration promotes sleep, and VIS depends on the genotype, sex, and time of day.

VIS Results in the Accrual of Sleep Credit and Does Not Require Light and the Circadian Clock

To examine whether increased sleep during vibration functions as normal sleep and contributes to the accumulation of sleep credit, we subjected iso31, CS, and CSx-iso31 females to $6 \mathrm{~h}$

Figure 1. Vibration Promotes Sleep in Flies

(A) Sleep profiles of iso31, CS, and CSx-iso31 females and males exposed to vibration for $24 \mathrm{~h}$ starting at Zeitgeber time (ZT) 0 . Sleep was assayed using singlebeam DAM monitors; $n=61-95$.

(B) Daytime and nighttime changes (vibration day versus baseline day) in sleep amount, sleep bout length, and sleep bouts of flies shown in (A).

(C) Video analysis of iso31 females. Behavior was manually scored, and time spent engaged in each behavior during the first hour of vibration starting at ZT 1 (vibration) or the corresponding hour on the previous day (baseline) is shown; $\mathrm{n}=32$. For bout duration, the line inside the box indicates the median, and the whiskers indicate $10 \%$ and $90 \%$ percentiles.

In this and subsequent figures, error bars indicate SEMs; ns, not significant, ${ }^{*} \mathrm{p}<0.05,{ }^{* *} \mathrm{p}<0.01$, and ${ }^{* * *} \mathrm{p}<0.001$. Paired Student's $\mathrm{t}$ test $(\Delta$ sleep and $\Delta$ bout number) or Wilcoxon matched-pairs signed rank test ( $\Delta$ bout duration) with Bonferroni correction (B); 1-way repeated-measures ANOVA followed by multiple pairwise comparisons with Bonferroni correction (C).

See also Figure S1. 


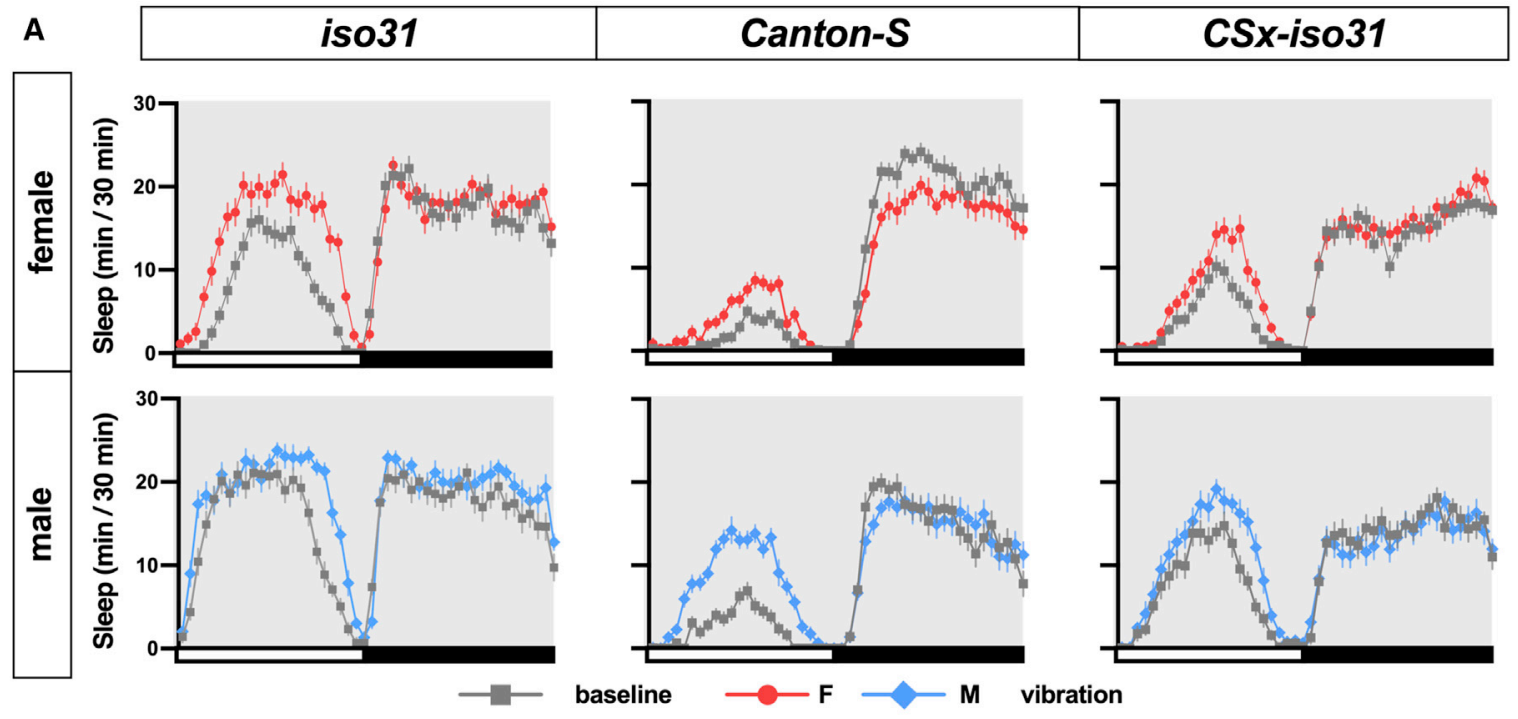

B
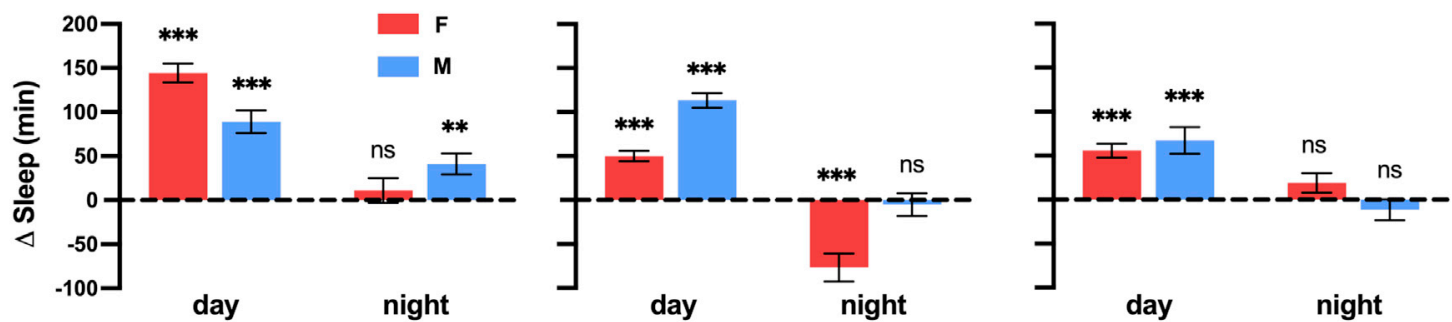

C
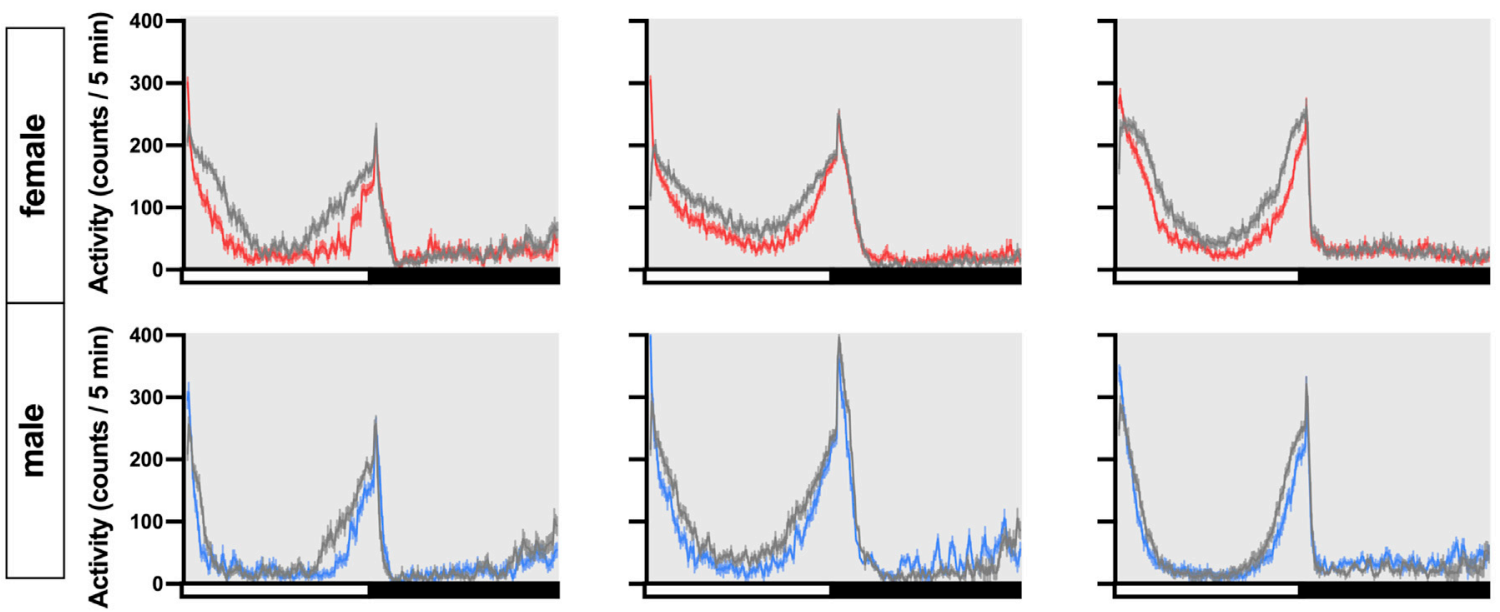

D
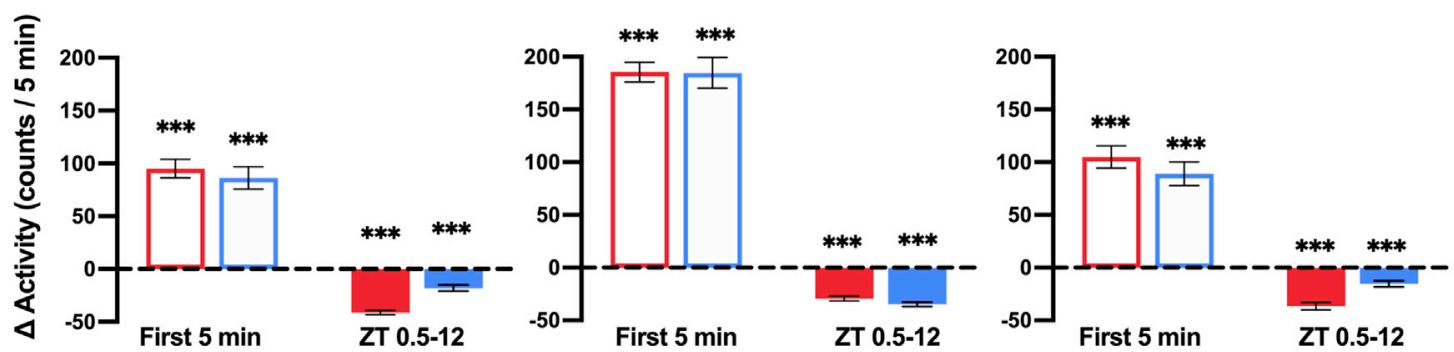
of vibration in the first half of the day. The end of vibration occurred at the peak of midday siesta when any decrease in sleep would be readily detectable. We observed a significant decrease in sleep, or negative rebound, in the $6 \mathrm{~h}$ after vibration following a substantial increase in sleep during the 6-h vibration in iso31 and CSx-iso31 females (Figures 3A and 3B). CS females exhibited significant VIS but little negative rebound. These data show that sleep gained during vibration can contribute to sleep credit and lead to reduced sleep after vibration, suggesting that VIS can substitute for normal sleep.

Since our results showed a greater increase in sleep during daytime compared to nighttime under LD conditions (Figures $1 \mathrm{~A}$ and $1 \mathrm{~B})$, we asked whether sleep increase by mechanical stimulation requires light or the circadian clock. To test this, we assayed sleep change during vibration in constant dark (DD) and constant light (LL) conditions. Flies exhibited clear VIS during the subjective day in DD (Figures $3 \mathrm{C}$ and $3 \mathrm{D}$ ), demonstrating that light is dispensable for the phenomenon. Whereas iso31 females exhibited small but significant negative rebound in DD, CS and CSx-iso31 females did not, suggesting that light and the genetic background influence negative rebound.

We next examined the role of the circadian clock in VIS. First, we assayed VIS in per ${ }^{01}$ arrhythmic mutants (Konopka and Benzer, 1971) in DD. per ${ }^{01}$ mutants exhibited modest but significant VIS and negative rebound (Figures S2A and S2B), suggesting that VIS does not require a functioning clock. Second, we measured VIS in LL, where wild-type flies become arrhythmic. All three strains exhibited significant VIS in LL (Figures $3 E$ and $3 F)$, confirming that the circadian clock is not required for VIS. In addition, all three strains exhibited significant negative rebound in LL, which supports the notion that VIS contributes to sleep credit accrual. Since CS females showed relatively modest VIS in all three conditions, we excluded them in subsequent experiments involving the vortexer.

Next, we assayed sleep in LL using multi-beam monitors and found similar patterns of VIS and negative rebound as with single-beam monitors (Figures $3 \mathrm{G}$ and $3 \mathrm{H}$ ). The only exception was that $C S$ females exhibited significant negative rebound measured using single-beam but not multi-beam monitors, which may be due to lower baseline sleep measured by multi-beam monitors. Combined inter-beam and intra-beam activity increased sharply during the first $5 \mathrm{~min}$ of vibration but gradually declined below the baseline level as vibration continued (Figures $3 \mathrm{I}$ and 3J). Activity levels increased beyond the baseline level but returned to pre-vibration levels a few hours after vibration ended. Similar changes were observed when inter-beam and intra-beam activity were analyzed separately (Figure S2C), confirming that vibration suppressed both local movements and locomotion across the monitor tubes. Collectively, our data demonstrate that VIS does not require the circadian clock and light and that sleep credit accumulates during VIS.

\section{VIS Reduces Sensory Responsiveness to Light}

One of the defining characteristics of sleep is increased arousal threshold (Campbell and Tobler, 1984). To determine how vibration affects arousability, we compared the probability of sleeping flies to awaken in response to light during periods of vibration and no vibration. We performed the assay in LL, in which flies sleep a moderate amount throughout the day, as it renders flies arrhythmic and thus eliminates the need to control for circadian fluctuations in arousability. We found that $1 \mathrm{~min}$ of extremely bright light ( $\sim 15,000$ lux $)$ superimposed on constant, moderate light ( 500 lux) can awaken $40 \%-65 \%$ of sleeping flies within 2 min under baseline (no vibration) conditions (Figure 4A). We, therefore, used bright light of varying durations $(1 \mathrm{~s}, 15 \mathrm{~s}$, and $1 \mathrm{~min}$ ) to measure sensory responsiveness in sleeping flies during vibration compared to no vibration. Vibration substantially reduced the responsiveness of flies to visual stimuli for iso31 and CSx-iso31 males and females at all stimulus durations except for $1 \mathrm{~s}$ light stimulation of iso31 males (Figure 4A). Spontaneous awakening in the absence of light pulses (i.e., 0 s pulse) was also reduced during vibration in iso31 females and CS $x$ iso31 males, but the greater effects of vibration in light pulse conditions suggest that sensory responsiveness is reduced by vibration. One minute of dark pulses was sufficient to awaken $>75 \%$ of sleeping flies (Figure S3A), showing that salient changes in the visual environment can reverse VIS. These results show that sleep during vibration is associated with reduced arousability relative to baseline sleep, which suggests that sleep during vibration is deeper than baseline sleep.

\section{Vibration Has Variable Effects on Short-Sleeping \\ Mutants}

We next asked whether genetic mutations that affect baseline sleep also influence VIS. We applied vibration for $24 \mathrm{~h}$ in LD to several short-sleeping mutants: sleepless (sss)/quiver, dopamine transporter (DAT), taranis (tara), and $n A C h R \alpha 4$ (Afonso et al., 2015; Koh et al., 2008; Kume et al., 2005; Shi et al., 2014). All of the mutant lines were outcrossed to the iso31 strain to minimize variability in genetic background. All of the mutant females exhibited reduced baseline sleep compared with control (iso31) flies (Figures $4 \mathrm{~B}$ and $\mathrm{S} 3 \mathrm{~B}$ ). $\operatorname{tara}^{\mathrm{s} 132}$ and $n A C h R \alpha 4^{\text {redeye (rye) }}$ mutants exhibited substantial VIS (Figures 4B and 4C), suggesting that the short-sleeping phenotype of some mutants can be rescued by mechanosensory stimulation. Interestingly, tara ${ }^{s 132}$ and $n A C h R \alpha 4^{r y e}$ exhibited greater nighttime sleep gain than control iso31 flies, suggesting that nighttime VIS in iso31 flies is limited by high baseline sleep. In contrast to $\operatorname{tara}^{\text {s132 }}$ and $n A C h$ $R \alpha 4^{r y e}$ mutants, $s s s^{P 1}$ and $D A T^{\text {fmn }}$ mutants exhibited little

Figure 2. Vibration Promotes Sleep and Produces Dynamic Changes in Locomotion and Local Movements

(A) Sleep profiles of iso31, CS, and CSx-iso31 females and males exposed to vibration for $24 \mathrm{~h}$ starting at ZT 0. Sleep was assayed using multi-beam DAM monitors; $n=46-62$.

(B) Daytime and nighttime changes in sleep amount of the flies shown in (A).

(C) Activity profiles in 5-min bins of the flies shown in (A).

(D) Activity changes during the first 5 min of vibration or ZT $0.5-12$ of flies shown in (A).

Paired Student's t test with Bonferroni correction (B and D). 


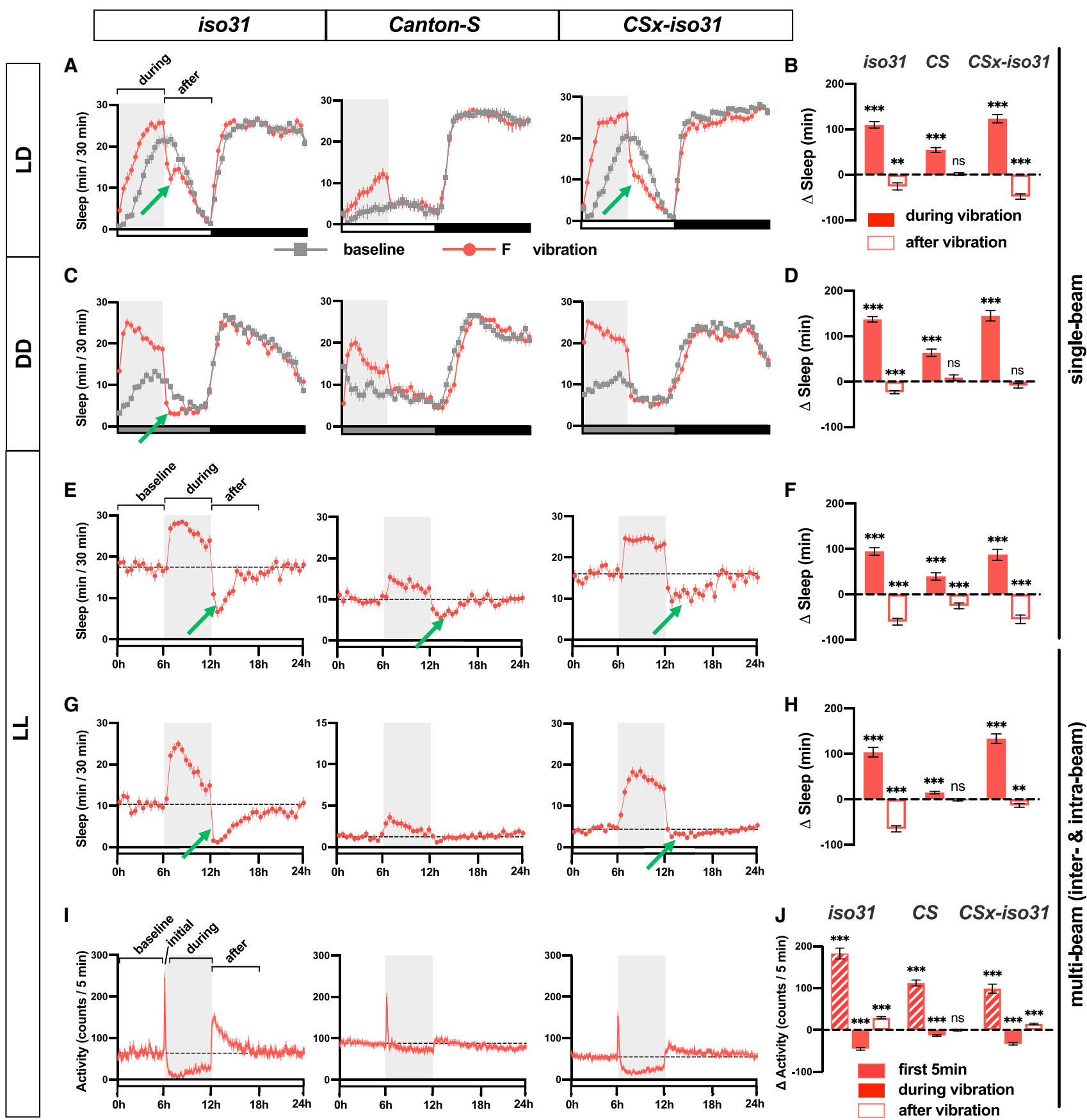

Figure 3. VIS Results in the Accrual of Sleep Credit and Does Not Require Light and the Circadian Clock

(A, C, E, and G) Sleep profiles of iso31, CS, and CSx-iso31 females in LD (A), DD (C), and LL (E and G), exposed to vibration for $6 \mathrm{~h}$ starting at ZT 0 or circadian time (CT) 0 . Single-beam monitors were used in (A), (C), and (E), whereas multi-beam monitors were used in $(G) ; n=46-92$. The gray box indicates the 6-h period of vibration. Green arrows point to negative rebound.

(B, D, F, and H) Sleep change during or after $6 \mathrm{~h}$ vibration relative to a $6-\mathrm{h}$ baseline period of flies shown in (A), (C), (E), and (G), respectively. (I) Activity profile of flies shown in (G). Both inter-beam and intra-beam movements in multi-beam monitors are included.

(J) Changes in activity count for $6 \mathrm{~h}$ before vibration (baseline), first $5 \mathrm{~min}$ (initial) or $0.5-6 \mathrm{~h}$ (during) of vibration, and $6 \mathrm{~h}$ after vibration (after).

Paired Student's t test with Bonferroni correction (B, D, F, and H), repeated-measures ANOVA followed by Dunnett's post hoc test relative to baseline (J). See also Figure S2. 
A

\begin{tabular}{|c|c|c|c|}
\hline \multicolumn{2}{|c|}{ iso31 } & \multicolumn{2}{c|}{ CSx-iso31 } \\
\hline female & male & female & male \\
\hline
\end{tabular}

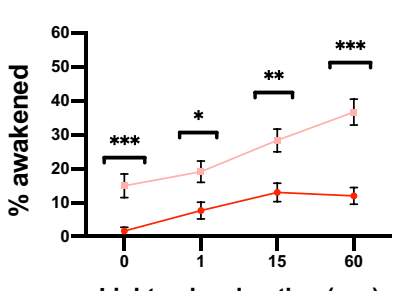

Light pulse duration (sec)

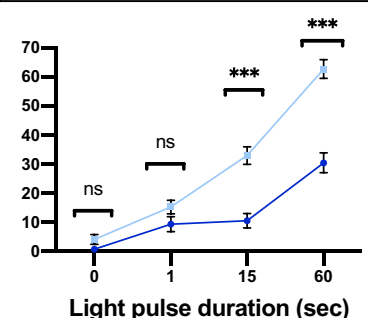

Light pulse duration (sec)

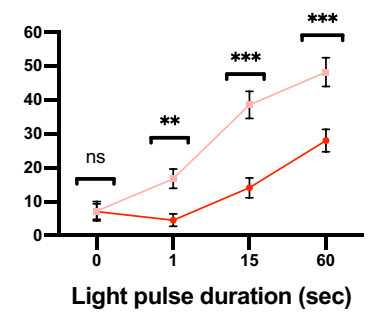

Light pulse duration (sec)

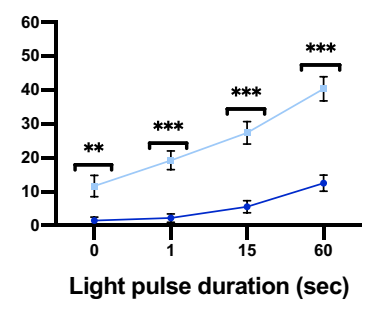

no vibration $\quad$ vibration
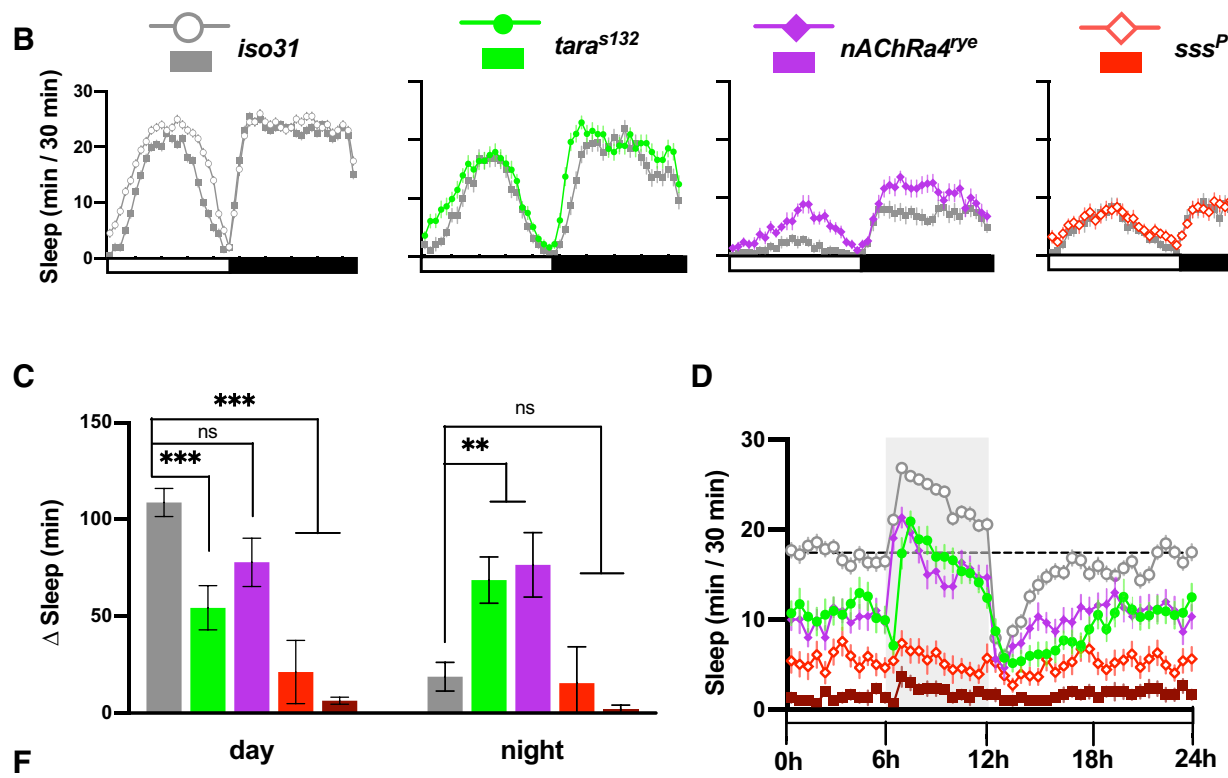

E

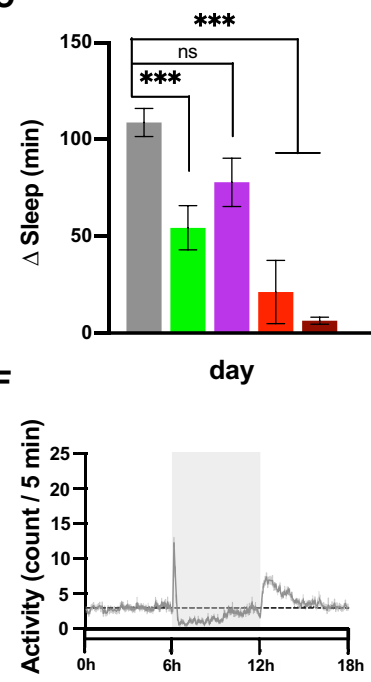

D
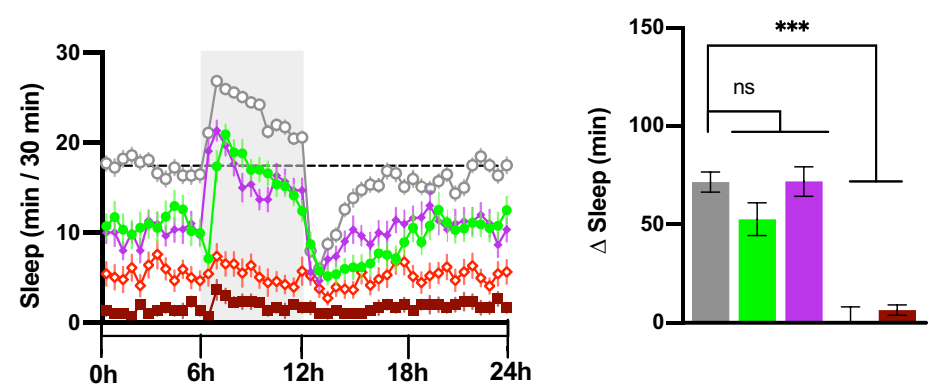

G

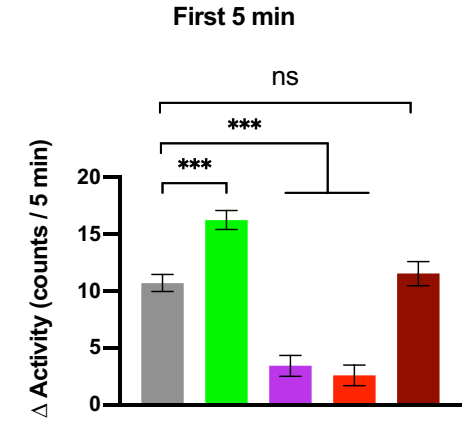

H

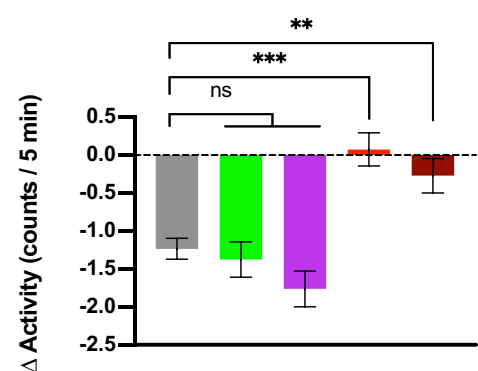

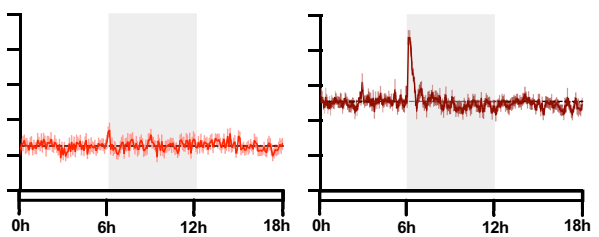

I

0-6h after vibration

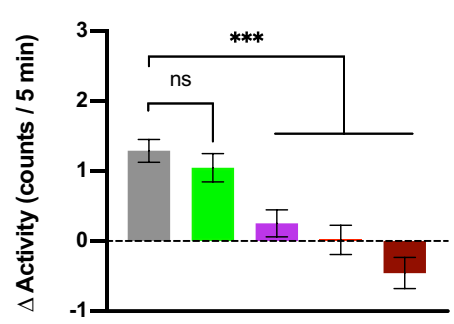


change in sleep during vibration (Figures 4B and 4C), confirming that the genetic background has a substantial impact on VIS.

To examine vibration-induced changes in activity in the sleep mutants, we subjected them to 6 -h vibration in LL, and sleep during vibration was compared to baseline sleep during the preceding $6 \mathrm{~h}$. All of the mutants exhibited reduced baseline sleep in LL (Figures 4D and S3B). As in LD, tara ${ }^{s 132}$ and $n A C h R \alpha 4^{r y e}$ mutants exhibited substantial VIS, whereas $s s s^{P 1}$ and $D A T^{f m n}$ mutants

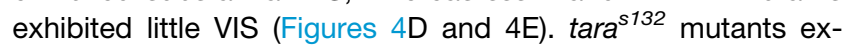
hibited a significant negative rebound, and $n A C h R \alpha 4^{\text {rye }}$ showed a brief negative rebound, which did not reach significance when computed over $6 \mathrm{~h}$ (Figures 4D and S3D). How much sleep credit accumulates during VIS likely depends on several factors, such as the rate of dissipation of sleep drive during sleep and the rate of dissipation of sleep credit during wakefulness, and our data suggest that these rates differ between $\operatorname{tara}^{\mathrm{s} 132}$ and $n A C h R \alpha 4^{\text {rye }}$ mutants.

Whereas tara ${ }^{5132}$ mutants showed a normal response to vibration (i.e., an initial increase in activity followed by a gradual decrease to a level below the baseline level), $n A C h R \alpha 4^{\text {rye }}$ mutants exhibited only a modest initial increase followed by a rapid decrease (Figures 4F-4H), which suggests distinct response kinetics in different genetic backgrounds. In contrast, $s s s^{P 1} \mathrm{mu}-$ tants did not show a noticeable change in locomotion during vibration, suggesting that the loss of sss may affect the sensory processing of vibration. Only control flies and tara ${ }^{s 132}$ mutants exhibited increased activity post-vibration (Figures 4F and 4I), consistent with the above result that they were the only ones to show significant negative rebound (Figure S3D). Interestingly, although $D A T^{f m n}$ mutants exhibited increased locomotion that gradually decreased over time, the activity level did not fall below the baseline level (Figures 4F-4I). DAT ${ }^{f m n}$ mutants harbor a genetic lesion in the DAT gene, which is expected to cause increased dopamine signaling and heightened arousal (Kume et al., 2005). It appears that $D A T^{f m n}$ mutants stay aroused during vibration despite normal sensory processing of vibration. These results suggest that dopaminergic arousal signals can counteract the sleep-promoting effects of vibration.

Habituation Learning Leads to Improved Sleep Induction in Successive Blocks of Vibration

To test whether habituation to vibration alters sleep induction, we presented iso31 and CSx-iso31 females with a series of $1-\mathrm{h}$ vibration training blocks interspersed with 1-h rest periods (Fig- ure 5A). Habituation can be distinguished from sensory adaptation or motor fatigue by stimulus specificity, namely a stimulus similar to but distinct from the habituated stimulus can restore response strength to pre-habituation levels (Rankin et al., 2009; Thompson and Spencer, 1966). To assess stimulus specificity, we compared continuous vibration to intermittent (2 min on, 2 min off) vibration. Since the two types of vibration were essentially the same, differing only in 2-min gaps, a recovery of pre-habituation response when switched to a new type of vibration would provide evidence of stimulus specificity. Flies were exposed to either four continuous-vibration blocks followed by one intermittent-vibration block or four intermittent-vibration blocks followed by one continuous-vibration block (Figure 5A). The experiment was performed in LL to remove circadian variation in the magnitude of VIS. Both continuous and intermittent vibration promoted sleep, and the sleep-promoting effects increased over the four blocks (Figures 5B and 5C). Continuous vibration was more effective in inducing sleep than intermittent vibration in the first block, as evidenced by greater sleep gain and shorter sleep latency, but the difference in sleep latency disappeared by the fourth block (Figures 5C and 5D).

Switching from continuous to intermittent vibration and vice versa allowed us to examine stimulus specificity. Sleep in flies switched from continuous to intermittent vibration on the fifth block was more similar to sleep in flies experiencing intermittent vibration for the first time than those experiencing it for the fourth time (Figures 5C and 5D). This finding demonstrates stimulus specificity in that training with continuous vibration did not generalize to intermittent vibration, even though the stimuli are essentially identical. Some stimulus generalization was observed, however, especially with respect to locomotor activity. Flies switched from continuous to intermittent vibration on the fifth block exhibited initial arousal responses (i.e., activity increase in the first $5 \mathrm{~min}$ of vibration) more similar to flies experiencing intermittent vibration for the fourth time than for the first time (Figures 5E and 5F). Switching from intermittent to continuous vibration did not show stimulus specificity, which may represent a ceiling effect since flies exposed to continuous vibration reached asymptotic levels of sleep amount and latency within the first hour of stimulation. Our finding of stimulus specificity suggests that habituation, but not sensory adaptation or motor fatigue, plays a major role in VIS.

Next, we tested whether repeated arousal responses to vibration onsets are sufficient for VIS. We presented flies with a brief

Figure 4. Sensory Responsiveness to Light Is Reduced during VIS, and Vibration Has Variable Effects on Short-Sleeping Mutants

(A) Percentage of sleeping flies that start moving within $2 \mathrm{~min}$ in response to bright light during periods of vibration or no vibration. iso31 and CSx-iso31 males and females were presented with bright light lasting $1 \mathrm{~s}, 15 \mathrm{~s}$, or $1 \mathrm{~min}$. The " $0 \mathrm{sec}$ " data represent spontaneous awakening in the absence of light stimuli; $\mathrm{n}=83-246$ from 48 flies.

(B) Sleep profile of females of indicated genotypes exposed to 6-h vibration in LD. The sleep mutants were outcrossed to the iso31 background at least 5 times; $\mathrm{n}=52-125$.

(C) Amount of daytime and nighttime sleep change during 6-h vibration compared to baseline (6 $\mathrm{h}$ before vibration) for flies shown in (B).

(D) Sleep profile of females of the indicated genotypes exposed to 6-h vibration in LL; $n=38-124$.

(E) Amount of sleep change during 6-h vibration compared to baseline for flies shown in (D).

(F) Activity profile of flies shown in (D).

(G-I) Activity change of flies shown in (B) during the first $5 \mathrm{~min}(\mathrm{G})$ or $0.5-6 \mathrm{~h}$ of vibration $(\mathrm{H})$, and $6 \mathrm{~h}$ after vibration (I).

Gray box indicates the 6 -h period of vibration. Dotted lines indicate average baseline sleep (D) or activity $(F)$. $\chi^{2}$ test with Bonferroni correction (A), Brown-Forsythe and Welch ANOVA followed by Dunnett's post hoc test relative to controls (C, E, and G-I).

See also Figure S3. 
A

continuous $\rightarrow$ intermittent

----- intermittent $\rightarrow$ continuous

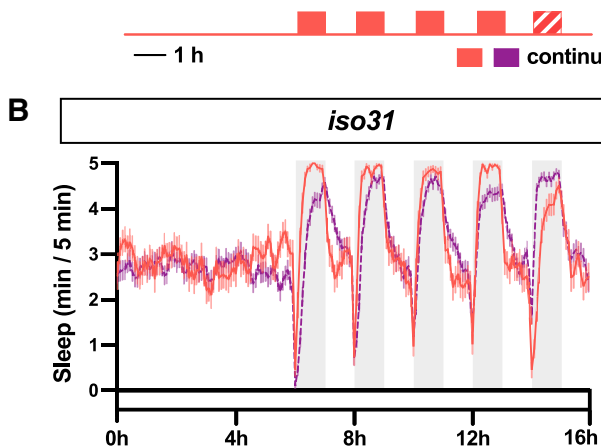

C
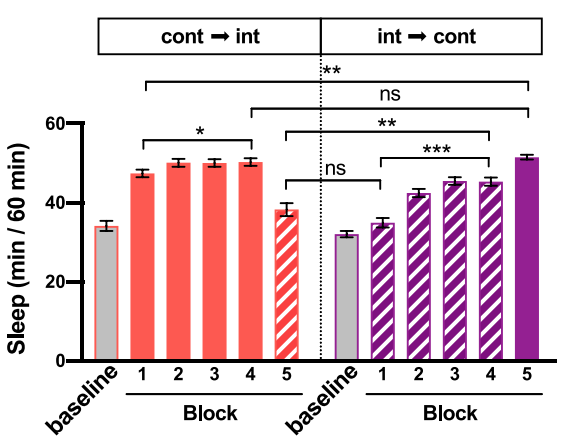

intermittent

Wa D W
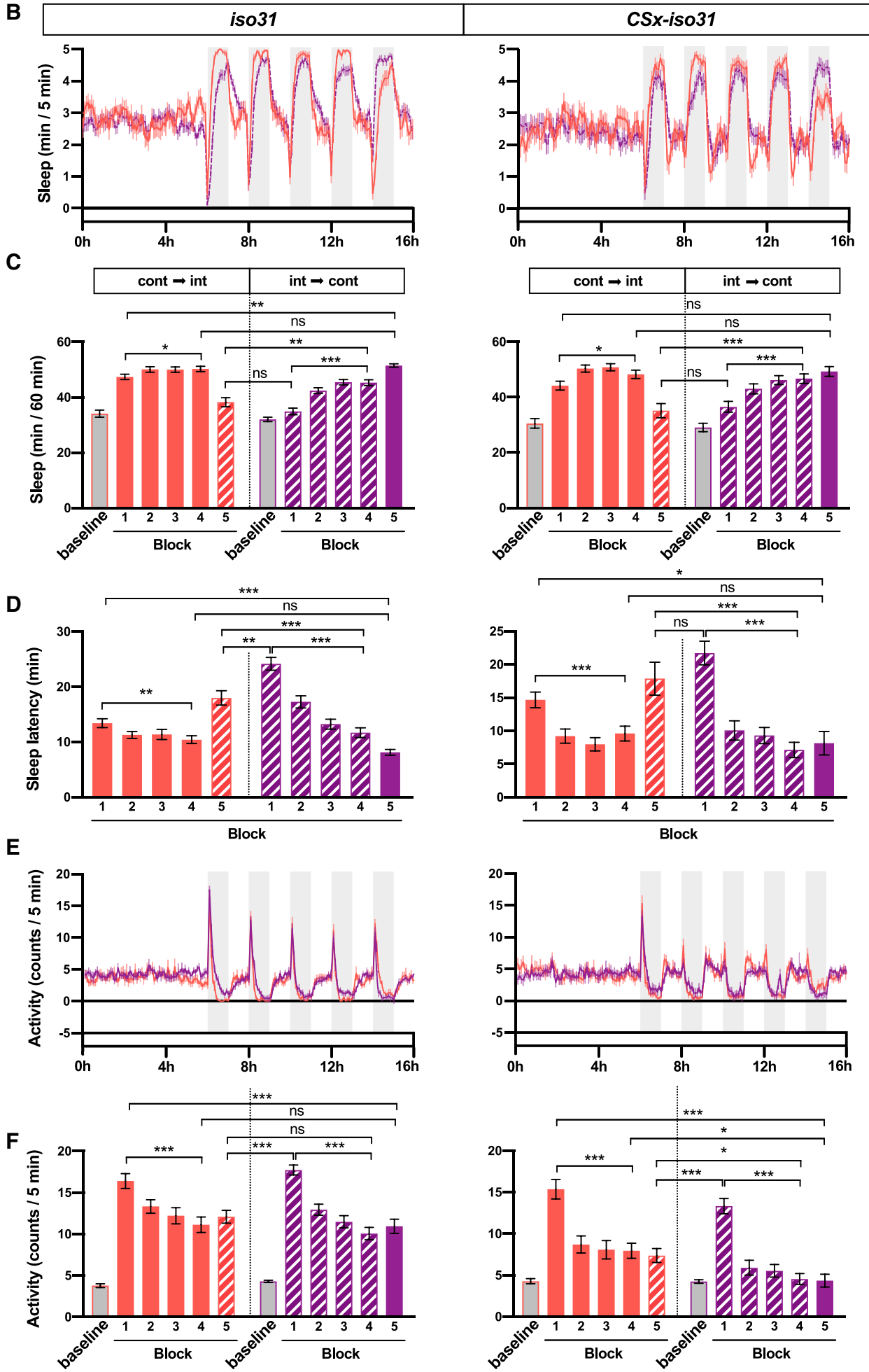

(legend on next page) 
(5-min) vibration every $2 \mathrm{~h}$ repeated 4 times (Figure S4A). We found that four brief vibration blocks were insufficient to induce sleep during the 1-h period following the vibration onset (Figures S4B and S4C). Although sleep latency decreased in iso31 females and activity decreased in both strains (Figures S4D$\mathrm{S} 4 \mathrm{~F}$ ), the changes were modest compared to those elicited by 1-h continuous or intermittent vibration (Figures 5D-5F). These findings suggest that VIS requires extended vibration and the arousal responses to vibration onsets are not its primary driver. Overall, our data demonstrate an improvement in sleep induction over multiple vibration sessions and suggest that habituation and consequent reduction in arousal contribute to VIS.

\section{VIS Is Mediated in Part by the Chordotonal Organs, the Antennae, and GABA Signaling}

To determine whether chordotonal neurons are responsible for vibration sensing, we genetically ablated chordotonal neurons using a chordotonal neuron driver, nan-GAL4 (Kim et al., 2003), and the cell death gene, head involution defective (hid) (Zhou et al., 1997). We observed a significantly lower amount of sleep increase in flies with ablated chordotonal neurons compared to genetic controls (Figures 6A and 6B), which suggests that chordotonal neurons constitute a major mediator of sleep induction by vibration. The small residual effects of vibration in the flies with ablated chordotonal neurons may reflect the contribution of other mechanosensory organs such as bristles. Thermogenetic activation of chordotonal neurons using dTrpA1 (Hamada et al., 2008) resulted in increased sleep (Figures 6C and 6D), suggesting that the activity of these neurons can contribute to sleep regulation. Several studies reported nan-Gal4 expression in chordotonal organs of the antennae, legs, and wing bases as well as a small number of neurons in the brain (Jourjine et al., 2016; Kim et al., 2003; Liu et al., 2007; Sehadova et al., 2009; Sun et al., 2009). As previously reported, we observed prominent nan-Gal4 expression in the antennal mechanosensory and motor center in the brain (Figure S5A), which presumably represents the nerve terminal of chordotonal organs in the antennae. Similarly, the ventral nerve cord expression pattern likely represents the nerve terminal of leg and wing base chordotonal organs. We detected nan-Gal4 expression in a small number of brain cells, but it was sporadic and variable (Figure S5A), and thus it is unlikely that expression in the central nervous system contributed significantly to the sleep effects of manipulating cells controlled by nan-Gal4.

To determine whether the antennae, which contain a subset of chordotonal organs, mediate sleep induction by vibration, we physically ablated the antennae of control flies and applied vibra- tion. Both iso31 and CSx-iso31 flies exhibited significantly reduced VIS in the absence of their antennae compared to their peers with intact antennae (Figures 6E and S5B). However, even without the antennae, iso31 flies showed significant VIS. These results show that antennae contribute to VIS and that their contribution depends on the genetic background.

To investigate the neural mechanisms downstream of the mechanosensory neurons, we next assessed VIS in flies with impaired GABA signaling. We examined the GABA signaling pathway because the habituation model proposes that habituation to monotonous stimuli leads to reduced arousal and increased sleep through a common inhibitory mechanism (Pavlov, 1927; Sokolov, 1963; Bohlin, 1971). To reduce GABA signaling, we knocked down glutamic acid decarboxylase 1 (Gad1), the gene encoding a nervous system-specific enzyme for GABA synthesis, by using Gad1-Gal4 (Hamasaka et al., 2005) and Gad1 shRNA. Knockdown of Gad1 led to a marked reduction in sleep induced by vibration, suggesting that an inhibitory mechanism is involved in VIS. Although Gad1 knockdown flies had increased baseline sleep relative to control flies (Figure $6 \mathrm{~F}$ ), the reduced VIS is unlikely to be due to a ceiling effect. Loss-of-function mutants in tyramine $\beta$ hydroxylase (Tbh), the gene encoding the key enzyme in octopamine synthesis (Monastirioti et al., 1996), exhibited robust VIS despite having a comparably high level of baseline sleep (Figures S5C and S5D). Overall, our results suggest that multiple sensory organs, including chordotonal neurons in the antennae and elsewhere in the body, convey mechanosensory information to the central sleep centers, in part through GABA signaling.

\section{Vibrations of a Wide Range of Frequencies Can Induce Sleep}

Since vortexers produce a complex pattern of rotational and translational motions that cannot be easily manipulated parametrically, we built an audio loudspeaker-based system that allowed us to produce vertical translational motions with independently controlled frequency and amplitude. Our speaker system was built based on a design previously used to study circadian entrainment by vibration (Simoni et al., 2014) (Figures 7A and S6A). We started with a combination of 20 and $200 \mathrm{~Hz}$ sinusoidal stimuli, as similar frequencies were used in the circadian entrainment experiments. We found that $24 \mathrm{~h}$ of vibration from the speaker system in LD had a profound effect on daytime sleep in male and female CS flies (Figures 7B and S6B). Vibration promoted sleep in iso31 and CSx-iso31 flies as well, although the effects were not as pronounced as in CS flies. As was the case with vibration generated by a vortexer, the effects of vibration on

Figure 5. Habituation Leads to Increased Sleep and Decreased Sleep Latency in Successive Blocks of Vibration

(A) Schematic diagram of the experimental design.

(B) Sleep profile of iso31 and CSx-iso31 females in the continuous $\rightarrow$ intermittent or intermittent $\rightarrow$ continuous condition. Gray boxes indicate the 1-h periods of vibration; $\mathrm{n}=48-124$.

(C) Sleep amount during the 6-h period before the first vibration block (baseline) and during 1-h vibration blocks (blocks 1-5) for flies shown in (B). Solid and striped bars represent continuous and intermittent stimulation, respectively.

(D) Sleep latency relative to the onset of vibration in each 1-h vibration block for flies shown in (B).

(E) Activity profile in 5 -min bins of flies shown in (B).

(F) Average activity during the initial 5 min of vibration in each 1-h vibration block.

Two-way repeated-measures ANOVA followed by selected post hoc tests with Bonferroni correction (C, D, and F).

See also Figure S4. 
A

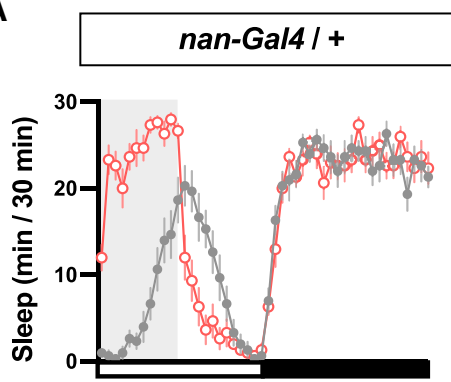

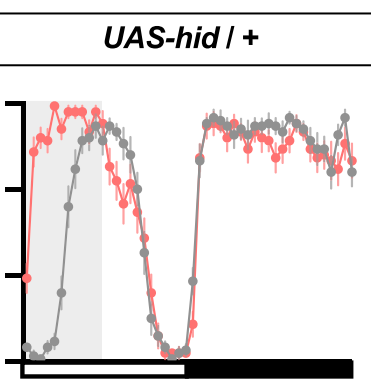

- baseline

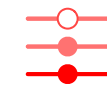

vibration

nan $>$ hid

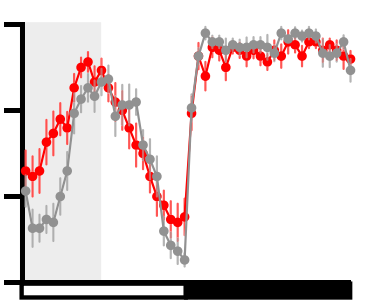

$31^{\circ} \mathrm{C}$

C
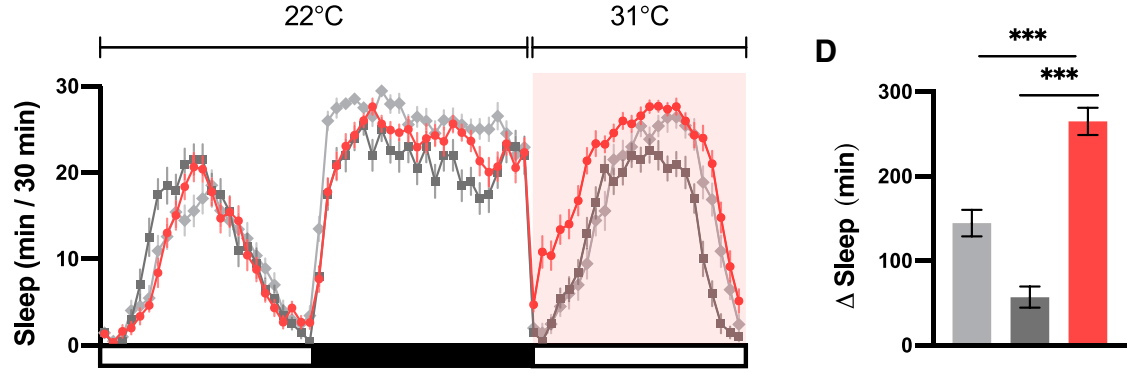

B

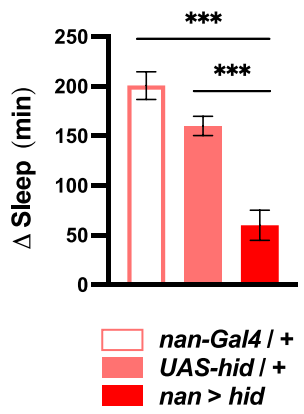

E

\begin{tabular}{|c|c|c|c|}
\hline \multicolumn{2}{|c|}{ iso31 } & \multicolumn{2}{c|}{ CSx-iso31 } \\
\hline intact antennae & no antennae & intact antennae & no antennae \\
\hline
\end{tabular}
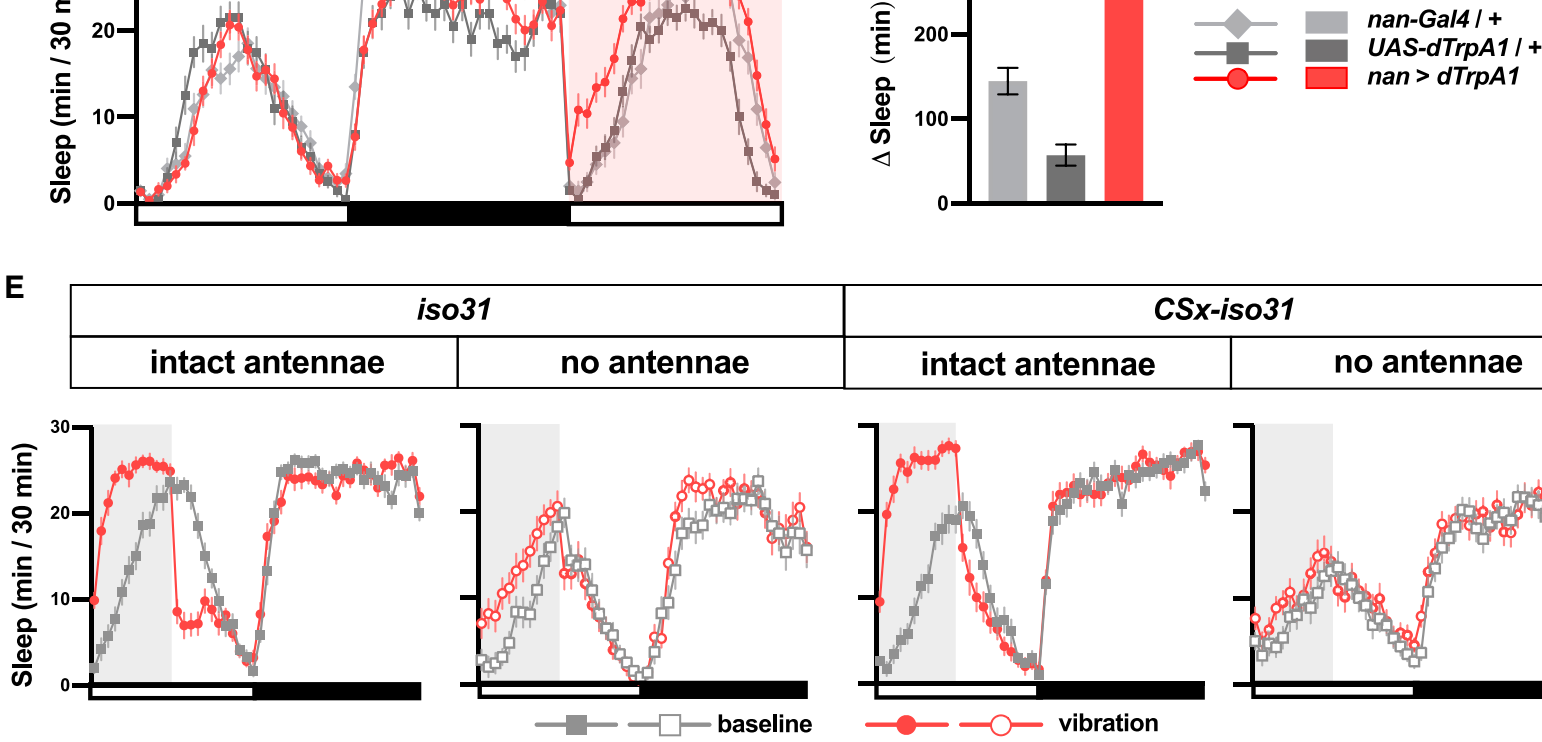

$\mathbf{F}$
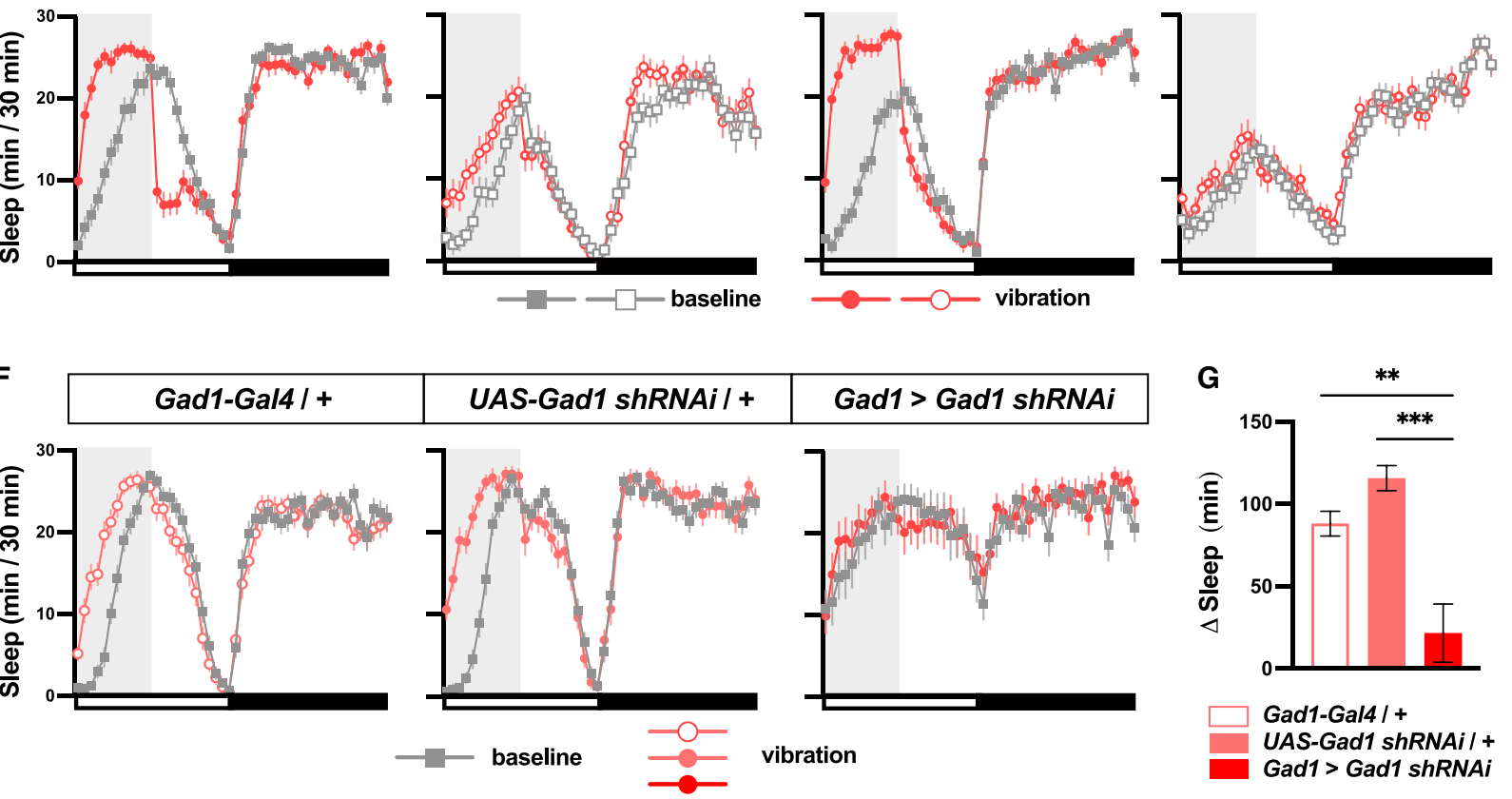

$-\mathrm{O}-$ vibration
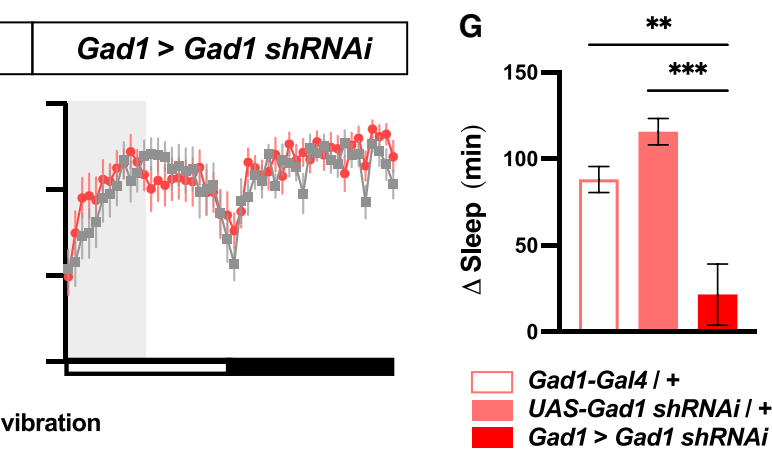

Figure 6. VIS Is Mediated in Part by the Chordotonal Organs and the Antennae

(A) Sleep profiles of females of indicated genotypes; $n=24$.

(B) Sleep change during or after $6 \mathrm{~h}$ vibration relative to a 6 -h period before vibration of flies shown in (A).

(C) Sleep profiles of female flies of indicated genotypes during baseline and during thermogenetic activation of chordotonal neurons. The orange box indicates the duration of exposure to high temperature; $n=29-41$.

(D) Change in daytime sleep amount when flies are exposed to high temperatures for flies shown in (C).

(E) Sleep profiles of iso31 and CSX-iso31 females with intact antennae or physically ablated antennae exposed to vibration for $6 \mathrm{~h}$ starting at ZT 0; $\mathrm{n}=37-52$. 
nighttime sleep were not as strong as those on daytime sleep. These data demonstrate that vibration generated by our speaker system can induce sleep in multiple control strains.

To determine the effects of vibration frequency on sleep, we applied vibrations at varying frequencies. Based on our data showing that VIS is more readily detectable in DD than in LD (Figure 3), we performed these experiments in a modified DD condition, in which it was dark except for 5-min light periods at Zeitgeber time (ZT) 0 and ZT 12. This lighting scheme, referred to as a "skeleton photoperiod" (Pittendrigh and Minis, 1964), allowed us to take advantage of greater vibration effects in darkness while minimizing the effects of variable circadian period lengths across individuals and genotypes. In addition to 20 and $200 \mathrm{~Hz}$ vibrations administered separately, we included 3 and $8 \mathrm{~Hz}$ vibrations. We used $3 \mathrm{~Hz}$ because it is close to the frequency range used in mammalian studies of rocking (Bayer et al., 2011; Kompotis et al., 2019; Perrault et al., 2019), and $8 \mathrm{~Hz}$ because a previous electrophysiological study found that the fly brain sometimes exhibits $7-10 \mathrm{~Hz}$ oscillations during sleep (Yap et al., 2017). Vibrations at all four frequencies were capable of inducing sleep, at least in some control strains (Figure 7C). Research in mice has found that within a range of $0.16-1.5 \mathrm{~Hz}$, acceleration determines the magnitude of sleep induced by rocking (Kompotis et al., 2019). Although the 8,20 , and $200 \mathrm{~Hz}$ stimuli in our study were comparable in acceleration (see Figure $7 \mathrm{C}$ legend), they produced different amounts of sleep gain. For example, the $200-\mathrm{Hz}$ vibration was less efficient at inducing sleep than the $20-\mathrm{Hz}$ vibration in all 3 strains, while vibration at $8 \mathrm{~Hz}$ had different effects depending on the genetic background (Figure 7C). Due to the limitations of the speaker system, the 3-Hz vibration we used had a much lower amplitude of acceleration than the vibrations at other frequencies. Overall, our data show that a variety of vibratory stimuli can induce sleep, and the magnitude of sleep gain is a function of the vibration frequency and the genetic background.

\section{DISCUSSION}

Our data establish that as in humans and mice, gentle mechanosensory stimulation can promote sleep in Drosophila. Flies showed increased activity and decreased sleep around lightdark transitions during $24 \mathrm{~h}$ vibration, indicating that they did not experience difficulty in locomotion and that the circadian arousal signal can counteract the sleep-promoting effects of vibration. Our observations that flies initially reacted to vibration with vigorous locomotion and that they can be awakened with salient changes in the visual environment also confirm that VIS is unrelated to other types of suppressed locomotion such as that induced by wind or fear in flies (Gibson et al., 2015; Yorozu et al., 2009) or tonic immobility in birds (Gallup, 1977). Previous studies have suggested that flies transition between lighter and deeper sleep stages during extended sleep bouts (Yap et al., 2017). Our results demonstrate that sleep during vibration is asso- ciated with reduced arousability, which suggests that vibration promotes deep sleep. Moreover, flies exhibited negative rebound, suggesting that excess sleep during vibration contributed to the accrual of sleep credit. Thus, VIS functions similarly to normal sleep in terms of its effect on sleep drive. An important future goal would be to determine whether VIS could provide other functions of sleep such as improved memory and longevity.

The habituation model of how sensory stimulation promotes sleep proposes that habituation to repetitive, unimportant stimuli leads to reduced arousal (Pavlov, 1927; Sokolov, 1963; Bohlin, 1971). According to the model, sensory inputs would result in increased sleep if they decreased arousal to a level below the baseline level, independent of the stimulus frequency. Our result that vibration of a wide range of frequency $(3-200 \mathrm{~Hz})$ can induce sleep supports the habituation model. Our findings that flies fall asleep faster and stay asleep longer over successive blocks of vibration and that this improvement does not generalize from continuous vibration to intermittent vibration further support the habituation model. Moreover, $D A T^{\mathrm{fmn}}$ mutants, which exhibit increased arousal, are resistant to the sleep-promoting effects of vibration, consistent with the view that reduced arousal is essential for sleep induction by sensory stimulation. Reduced GABA signaling also renders flies resistant to the effects of vibration, implicating inhibitory mechanisms in VIS. We found that sleep amount and latency show stimulus specificity while activity count does not, which resembles several studies demonstrating that different response components exhibit distinct habituation kinetics and are controlled by distinct molecules and neural circuits in zebrafish and Caenorhabditis elegans (Flavell et al., 2013; McDiarmid et al., 2019; Randlett et al., 2019). These findings led to a recent proposal that habituation is more than simply learning to ignore and that it allows organisms to switch between alternative behaviors, depending on the context (McDiarmid et al., 2019). As flies learn to recognize a specific vibratory stimulus as non-threatening, they may choose to sleep in its presence.

An alternative model of the sleep-promoting effects of sensory stimulation is that sensory inputs can synchronize cortical activity and boost sleep slow waves (Bellesi et al., 2014; Perrault et al., 2019). Although it is unknown whether there are brain-wide oscillations within the delta frequency range during sleep in Drosophila, the previous observation that the fly brain exhibits $7-10 \mathrm{~Hz}$ oscillations during some periods of sleep (Yap et al., 2017) raises the possibility that $8 \mathrm{~Hz}$ stimulation may promote sleep in part through the synchronization mechanism. Overall, our data are consistent with the habituation model, but synchronization of brain activity may also contribute to VIS under specific stimulus conditions.

Previous studies have shown various effects of vibration on fly behavior (Hill and Wessel, 2016). For instance, daily cycles of vibration and silence can entrain the circadian clock (Simoni et al., 2014), and male courtship songs (i.e., airborne acoustic vibrations) play a critical role in courtship behavior (Aranha and Vasconcelos, 2018; Yamamoto and Koganezawa, 2013). Males

(F) Sleep profiles of females of indicated genotypes. Flies in this experiment were exposed to a combination of 20 and $200 \mathrm{~Hz}$ vibration generated by a custombuilt speaker system described in Figure 7. All prior experiments used a vortexer to generate vibration stimuli; $\mathrm{n}=19-42$.

(G) Sleep change during or after $6 \mathrm{~h}$ vibration relative to a 6 -h period before vibration of flies shown in (F).

The gray boxes indicate the 6-h period of vibration. One-way Brown-Forsythe and Welch ANOVA followed by Dunnett's (B, D, and G) post hoc test. See also Figure S5. 
A

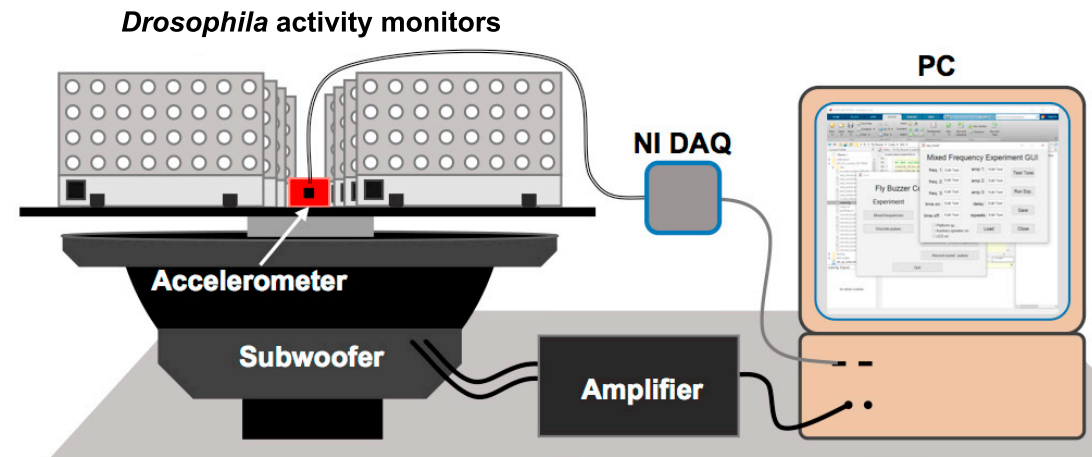

B

\begin{tabular}{|c|c|c|}
\hline \multicolumn{3}{|c|}{ LD, $20 \mathrm{~Hz}+200 \mathrm{~Hz}$} \\
\hline iso31 & Canton-S & CSx-iso31 \\
\hline
\end{tabular}
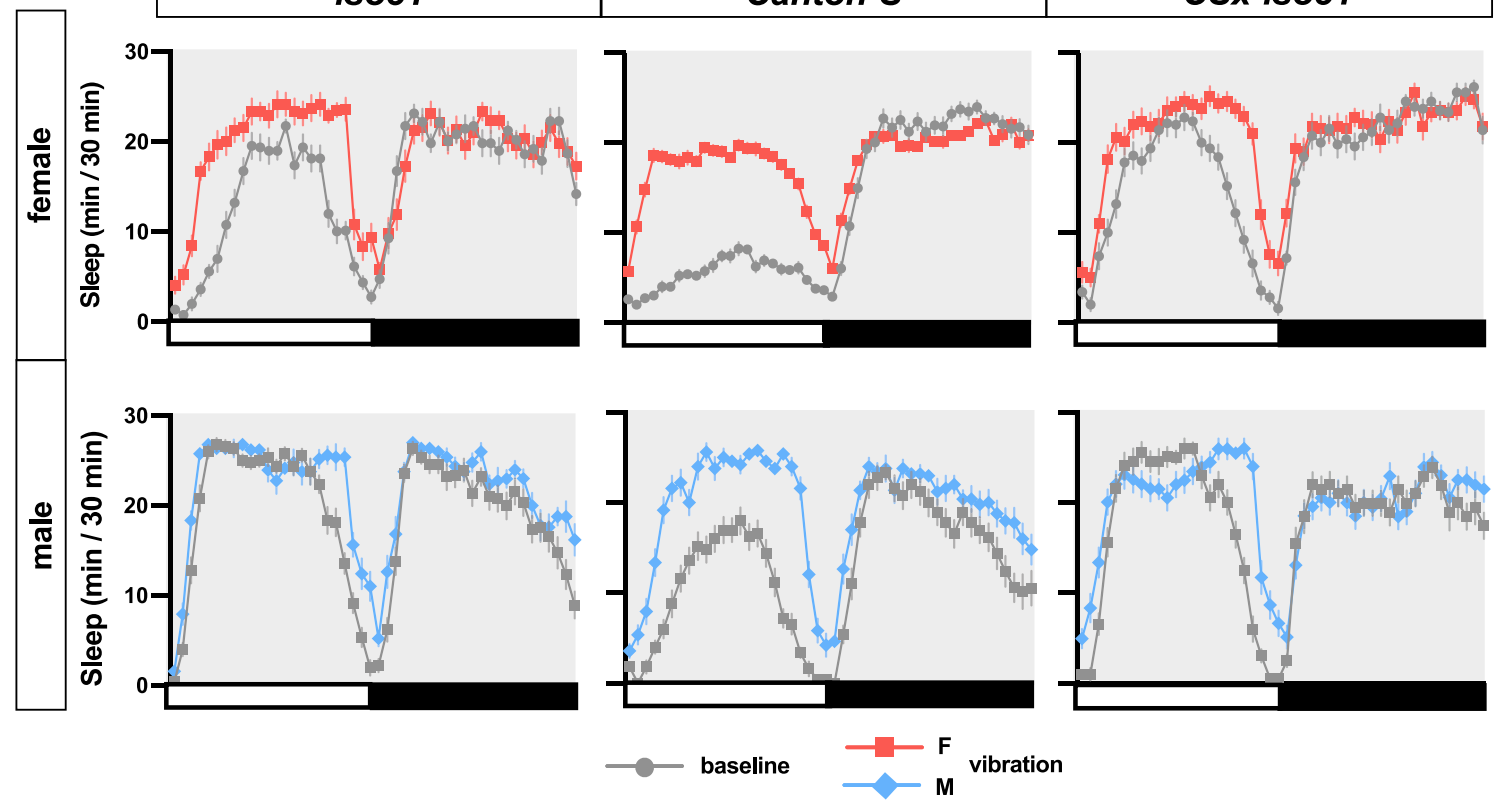

C

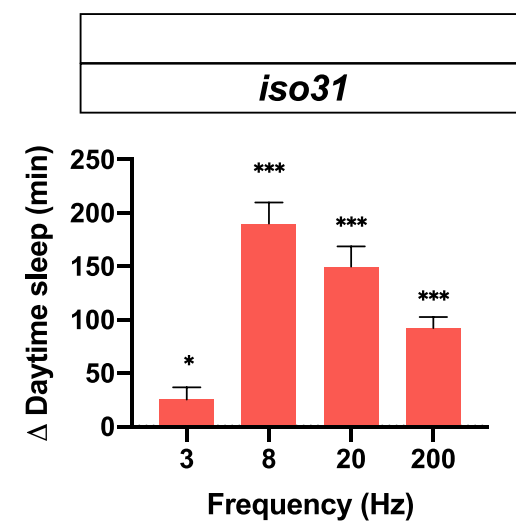

\section{DD+, individual frequency}

\begin{tabular}{|c|c|}
\hline Canton-S & CSx-iso31 \\
\hline
\end{tabular}
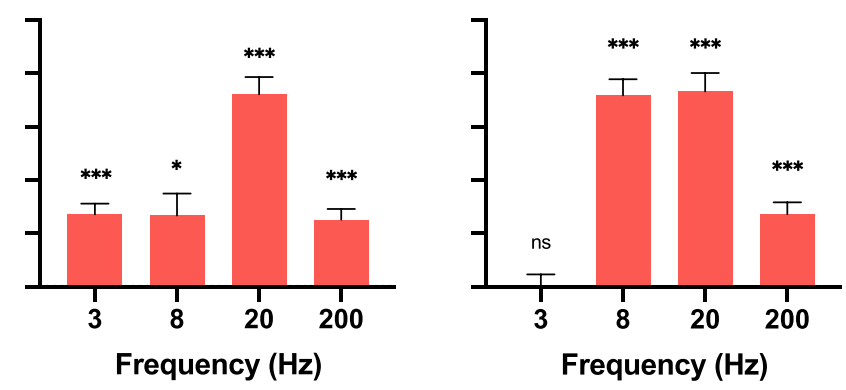

Figure 7. VIS Depends on the Stimulus Frequency and Genetic Background

(A) Schematic representation of speaker system. Fly activity monitors were fastened to a platform, which was glued to the cone of a 15 -in marine subwoofer. An accelerometer mounted on the platform was used to measure the amplitude of vibration via a data acquisition device (NI DAQ). See Method Details for additional details. 
also generate substrate-borne vibrations during courtship by shaking their abdomen, and females stop walking and become receptive to courtship when they sense the vibrations (Fabre et al., 2012; Mazzoni et al., 2013). Vibrational communication has been documented in other insect species as well (Cocroft and Rodríguez, 2005; Virant-Doberlet and Cokl, 2004). Flies likely monitor intentional and incidental vibrations produced by other flies and animals to determine their significance. In addition to the vibrational signals from other animals, the natural environment provides other vibratory cues such as a gentle breeze on a leaf. Falling asleep when there is repetitive mechanical stimulation may provide an as yet unidentified adaptive advantage. It is possible that monotonous gentle vibration from a breeze or a group of flies signals a relatively safe environment for sleep.

Our data suggest that multiple sensory organs are involved in sleep induction by vibration in Drosophila, including chordotonal organs in the antennae and the rest of the body. Previous studies suggest that the effects of substrate vibration on courtship and circadian entrainment are mediated by chordotonal organs ( $\mathrm{Fa}$ bre et al., 2012; Simoni et al., 2014). The latter study found that the antennae are dispensable for circadian entrainment by a vibration of a specific combination of frequencies, but the antennae may be able to contribute to circadian entrainment when vibrations of different frequencies are involved. Similarly, although a recent study using rhythmic horizontal movements at $0.25-1.5 \mathrm{~Hz}$ found that the vestibular otolithic organs mediate the effects of rocking on sleep in mice (Kompotis et al., 2019), multiple mechanosensory organs may also mediate the effects of mechanosensory stimuli on sleep in mammals.

Most studies of the effects of sensory stimulation on sleep have used mechanosensory stimuli such as rocking or acoustic stimulation (Bayer et al., 2011; Bohlin, 1971; Kompotis et al., 2019; Perrault et al., 2019; Tononi et al., 2010), and whether stimulation in other sensory modalities also influences sleep is an interesting and unresolved question. A few studies involving olfactory stimulation in humans and rats found variable results (Sano et al., 1998; Goel et al., 2005; Tononi et al., 2010), which may be due to differences in the odorants and how they were administered. Drosophila studies, given the relative ease of high-throughput analysis, may help address the question of the influence of olfactory and other non-mechanosensory stimuli on sleep. Regardless of whether non-mechanosensory stimulation can also promote sleep, sleep induction by sensory stimulation in flies provides a valuable platform for studying the neural and molecular mechanisms of sleep regulation.

\section{STAR $\star$ METHODS}

Detailed methods are provided in the online version of this paper and include the following:
- KEY RESOURCES TABLE

- RESOURCE AVAILABILITY

○ Lead Contact

- Material Availability

- Data and Code Availability

- EXPERIMENTAL MODEL AND SUBJECT DETAILS

○ Fly Stocks

- METHOD DETAILS

O Sleep Analysis

O Generation of Vibration Stimuli

$\bigcirc$ Video Recording and Quantification

O Analysis of Sensory Responsiveness

- Immunohistochemistry

O Antennae Ablation

O Transgenic Fly Lines

- QUANTIFICATION AND STATISTICAL ANALYSIS

\section{SUPPLEMENTAL INFORMATION}

Supplemental Information can be found online at https://doi.org/10.1016/j. celrep.2020.108462.

\section{ACKNOWLEDGMENTS}

We thank Drs. Amita Sehgal and Kazuhiko Kume and the Bloomington Stock Center for fly stocks; Dr. Gui-Shuang Ying for advice on the statistical analysis; Dr. Bill Joiner for the SleepLab software; and Jennifer Wilson, Dr. Matthew Kayser, and members of the Koh lab for helpful suggestions for improving the manuscript. This work was supported by grants from the National Institutes of Health (R01NS086887, to K.K., and R01NS084835, to C.F.-Y.) and funds from Thomas Jefferson University (Jefferson Center for Synaptic Biology award, to K.K.).

\section{AUTHOR CONTRIBUTIONS}

Conceptualization \& Methodology, A.O.-C., S.I., and K.K. Investigation, A.O.C., S.I., J.R.B., and A.C. Resources, P.D.M. and C.F.-Y. Writing - Original Draft, K.K. Writing - Review \& Editing, A.O.-C., S.I., J.R.B., P.D.M., A.C., and C.F.-Y. Funding Acquisition \& Supervision, K.K. and C.F.-Y.

\section{DECLARATION OF INTERESTS}

The authors declare no competing interests.

Received: April 14, 2020

Revised: September 11, 2020

Accepted: November 10, 2020

Published: December 1, 2020

\section{REFERENCES}

Afonso, D.J.S., Liu, D., Machado, D.R., Pan, H., Jepson, J.E.C., Rogulja, D. and Koh, K. (2015). TARANIS Functions with Cyclin A and Cdk1 in a Novel Arousal Center to Control Sleep in Drosophila. Curr. Biol. 25, 1717-1726.

(B) Sleep profiles of iso31, CS, and CSx-iso31 females and males exposed to a combination of 20 and $200 \mathrm{~Hz}$ vibrations for $24 \mathrm{~h}$ in LD starting at ZT 0 . The amplitude of acceleration (half the difference between peak and trough) was $22.68 \mathrm{~m} / \mathrm{s}^{2} ; \mathrm{n}=43-108$.

(C) Daytime sleep change relative to baseline day of CS, iso31, and CSx-iso31 females exposed to 24-h vibration of 3, 8, 20 , and $200 \mathrm{~Hz}$. Sleep assay was performed in a "DD+" condition (i.e., darkness except for 5 -min light pulses at ZT 0 and ZT 12). The amplitude of acceleration of $3,8,20$, and $200 \mathrm{~Hz}$ vibrations was $2.43,15.78,13.58$, and $14.56 \mathrm{~m} / \mathrm{sec}^{2}$, respectively; $\mathrm{n}=51-64$.

Paired Student's $t$ test with Bonferroni correction ( $B$ and $C$ ).

See also Figure S6. 
Albert, J.T., and Göpfert, M.C. (2015). Hearing in Drosophila. Curr. Opin. Neurobiol. 34, 79-85.

Aranha, M.M., and Vasconcelos, M.L. (2018). Deciphering Drosophila female innate behaviors. Curr. Opin. Neurobiol. 52, 139-148.

Bayer, L., Constantinescu, I., Perrig, S., Vienne, J., Vidal, P.P., Mühlethaler, M., and Schwartz, S. (2011). Rocking synchronizes brain waves during a short nap. Curr. Biol. 21, R461-R462.

Bellesi, M., Riedner, B.A., Garcia-Molina, G.N., Cirelli, C., and Tononi, G. (2014). Enhancement of sleep slow waves: underlying mechanisms and practical consequences. Front. Syst. Neurosci. 8, 208.

Bohlin, G. (1971). Monotonous stimulation, sleep onset and habituation of the orienting reaction. Electroencephalogr. Clin. Neurophysiol. 31, 593-601.

Campbell, S.S., and Tobler, I. (1984). Animal sleep: a review of sleep duration across phylogeny. Neurosci. Biobehav. Rev. 8, 269-300.

Chen, C., Schaeffer, L.V., Guo, M., and Hay, B. (2007). A Synthetic MaternalEffect Selfish Genetic Element Drives Population Replacement in Drosophila. Science 316, 597-600.

Cocroft, R.B., and Rodríguez, R.L. (2005). The behavioral ecology of insect vibrational communication. Bioscience 55, 323-334.

Delmas, P., Hao, J., and Rodat-Despoix, L. (2011). Molecular mechanisms of mechanotransduction in mammalian sensory neurons. Nat. Rev. Neurosci. 12, 139-153.

Ekdale, E.G. (2016). Form and function of the mammalian inner ear. J. Anat. 228, 324-337.

Fabre, C.C.G., Hedwig, B., Conduit, G., Lawrence, P.A., Goodwin, S.F., and Casal, J. (2012). Substrate-borne vibratory communication during courtship in Drosophila melanogaster. Curr. Biol. 22, 2180-2185.

Flavell, S.W., Pokala, N., Macosko, E.Z., Albrecht, D.R., Larsch, J., and Bargmann, C.I. (2013). Serotonin and the neuropeptide PDF initiate and extend opposing behavioral states in C. elegans. Cell 154, 1023-1035.

Gallup, G.D. (1977). Tonic Immobility: the Role of Fear and Predation. Psychol. Rec. 27, 41-61.

Garbe, D.S., Bollinger, W.L., Vigderman, A., Masek, P., Gertowski, J., Sehgal, A., and Keene, A.C. (2015). Context-specific comparison of sleep acquisition systems in Drosophila. Biol. Open 4, 1558-1568.

Gibson, W.T., Gonzalez, C.R., Fernandez, C., Ramasamy, L., Tabachnik, T., Du, R.R., Felsen, P.D., Maire, M.R., Perona, P., and Anderson, D.J. (2015). Behavioral responses to a repetitive visual threat stimulus express a persistent state of defensive arousal in Drosophila. Curr. Biol. 25, 1401-1415.

Goel, N., Kim, H., and Lao, R.P. (2005). An olfactory stimulus modifies nighttime sleep in young men and women. Chronobiol. Int. 22, 889-904.

Hamada, F.N., Rosenzweig, M., Kang, K., Pulver, S.R., Ghezzi, A., Jegla, T.J., and Garrity, P.A. (2008). An internal thermal sensor controlling temperature preference in Drosophila. Nature 454, 217-220.

Hamasaka, Y., Wegener, C., and Nässel, D.R. (2005). GABA modulates Drosophila circadian clock neurons via GABAB receptors and decreases in calcium. J. Neurobiol. 65, 225-240.

Hill, P.S.M., and Wessel, A. (2016). Biotremology. Curr. Biol. 26, R187-R191. Huber, R., Hill, S.L., Holladay, C., Biesiadecki, M., Tononi, G., and Cirelli, C. (2004). Sleep homeostasis in Drosophila melanogaster. Sleep 27, 628-639.

Joiner, W.J. (2016). Unraveling the Evolutionary Determinants of Sleep. Curr. Biol. 26, R1073-R1087.

Jourjine, N., Mullaney, B.C., Mann, K., and Scott, K. (2016). Coupled Sensing of Hunger and Thirst Signals Balances Sugar and Water Consumption. Cell $166,855-866$.

Kim, J., Chung, Y.D., Park, D.Y., Choi, S., Shin, D.W., Soh, H., Lee, H.W., Son, W., Yim, J., Park, C.S., et al. (2003). A TRPV family ion channel required for hearing in Drosophila. Nature 424, 81-84.

Koh, K., Joiner, W.J., Wu, M.N., Yue, Z., Smith, C.J., and Sehgal, A. (2008). Identification of SLEEPLESS, a Sleep-Promoting Factor. Science 321 , 372-376.
Kompotis, K., Hubbard, J., Emmenegger, Y., Perrault, A., Mühlethaler, M. Schwartz, S., Bayer, L., and Franken, P. (2019). Rocking Promotes Sleep in Mice through Rhythmic Stimulation of the Vestibular System. Curr. Biol. 29, 392-401.e4.

Konopka, R.J., and Benzer, S. (1971). Clock mutants of Drosophila melanogaster. Proc. Natl. Acad. Sci. USA 68, 2112-2116.

Korner, A.F., Guilleminault, C., Van den Hoed, J., and Baldwin, R.B. (1978). Reduction of sleep apnea and bradycardia in preterm infants on oscillating water beds: a controlled polygraphic study. Pediatrics 61, 528-533.

Kume, K., Kume, S., Park, S.K., Hirsh, J., and Jackson, F.R. (2005). Dopamine is a regulator of arousal in the fruit fly. J. Neurosci. 25, 7377-7384.

Liu, L., Li, Y., Wang, R., Yin, C., Dong, Q., Hing, H., Kim, C., and Welsh, M.J. (2007). Drosophila hygrosensation requires the TRP channels water witch and nanchung. Nature 450, 294-298.

Mazzoni, V., Anfora, G., and Virant-Doberlet, M. (2013). Substrate vibrations during courtship in three Drosophila species. PLOS ONE 8, e80708.

McDiarmid, T.A., Yu, A.J., and Rankin, C.H. (2019). Habituation Is More Than Learning to Ignore: Multiple Mechanisms Serve to Facilitate Shifts in Behavioral Strategy. BioEssays 41, e1900077.

Monastirioti, M., Linn, C.E.J., Jr., and White, K. (1996). Characterization of Drosophila tyramine beta-hydroxylase gene and isolation of mutant flies lacking octopamine. J. Neurosci. 16, 3900-3911.

Pavlov, I.P. (1927). Conditioned Reflexes (London: Oxford University Press).

Perrault, A.A., Khani, A., Quairiaux, C., Kompotis, K., Franken, P., Muhlethaler, M., Schwartz, S., and Bayer, L. (2019). Whole-Night Continuous Rocking Entrains Spontaneous Neural Oscillations with Benefits for Sleep and Memory. Curr. Biol. 29, 402-411.e3.

Pittendrigh, C.S., and Minis, D.H. (1964). The Entrainment of Circadian Oscillations by Light and Their Role as Photoperiodic Clocks. Am. Nat. 98, 261-294.

Randlett, O., Haesemeyer, M., Forkin, G., Shoenhard, H., Schier, A.F., Engert, F., and Granato, M. (2019). Distributed plasticity drives visual habituation learning in larval zebrafish. Curr. Biol. 29, 1337-1345.e4.

Rankin, C.H., Abrams, T., Barry, R.J., Bhatnagar, S., Clayton, D.F., Colombo, J., Coppola, G., Geyer, M.A., Glanzman, D.L., Marsland, S., et al. (2009). Habituation revisited: an updated and revised description of the behavioral characteristics of habituation. Neurobiol. Learn. Mem. 92, 135-138.

Ryder, E., Blows, F., Ashburner, M., Bautista-Llacer, R., Coulson, D., Drummond, J., Webster, J., Gubb, D., Gunton, N., Johnson, G., et al. (2004). The DrosDel collection: a set of P-element insertions for generating custom chromosomal aberrations in Drosophila melanogaster. Genetics 167, 797-813.

Sano, A., Sei, H., Seno, H., Morita, Y., and Moritoki, H. (1998). Influence of cedar essence on spontaneous activity and sleep of rats and human daytime nap. Psychiatry Clin. Neurosci. 52, 133-135.

Sehadova, H., Glaser, F.T., Gentile, C., Simoni, A., Giesecke, A., Albert, J.T., and Stanewsky, R. (2009). Temperature entrainment of Drosophila's circadian clock involves the gene nocte and signaling from peripheral sensory tissues to the brain. Neuron 64, 251-266.

Shi, M., Yue, Z., Kuryatov, A., Lindstrom, J.M., and Sehgal, A. (2014). Identification of Redeye, a new sleepregulating protein whose expression is modulated by sleep amount. eLife 3, e01473.

Simoni, A., Wolfgang, W., Topping, M.P., Kavlie, R.G., Stanewsky, R., and Albert, J.T. (2014). A Mechanosensory Pathway to the Drosophila Circadian Clock. Science 343, 525-528.

Sokolov, E.N. (1963). Perception and the Conditioned Reflex (Oxford: Pergamon Press).

Sun, Y., Liu, L., Ben-Shahar, Y., Jacobs, J.S., Eberl, D.F., and Welsh, M.J. (2009). TRPA channels distinguish gravity sensing from hearing in Johnston's organ. Proc. Natl. Acad. Sci. USA 106, 13606-13611.

Thompson, R.F., and Spencer, W.A. (1966). Habituation: a model phenomenon for the study of neuronal substrates of behavior. Psychol. Rev. 73, 16-43. Tononi, G., Riedner, B.A., Hulse, B.K., Ferrarelli, F., and Sarasso, S. (2010). Enhancing sleep slow waves with natural stimuli. Medicamundi 54, 73-79. 
Tuthill, J.C., and Wilson, R.I. (2016). Mechanosensation and Adaptive Motor Control in Insects. Curr. Biol. 26, R1022-R1038.

Virant-Doberlet, M., and Cokl, A. (2004). Vibrational communication in insects. Neotrop. Entomol. 33, 121-134.

Yamamoto, D., and Koganezawa, M. (2013). Genes and circuits of courtship behaviour in Drosophila males. Nat. Rev. Neurosci. 14, 681-692.

Yap, M.H.W., Grabowska, M.J., Rohrscheib, C., Jeans, R., Troup, M., Paulk, A.C., van Alphen, B., Shaw, P.J., and van Swinderen, B. (2017). Oscillatory brain activity in spontaneous and induced sleep stages in flies. Nat. Commun. 8, 1815.

Yorozu, S., Wong, A., Fischer, B.J., Dankert, H., Kernan, M.J., Kamikouchi, A. Ito, K., and Anderson, D.J. (2009). Distinct sensory representations of wind and near-field sound in the Drosophila brain. Nature 458, 201-205.

Zhou, L., Schnitzler, A., Agapite, J., Schwartz, L.M., Steller, H., and Nambu, J.R. (1997). Cooperative functions of the reaper and head involution defective genes in the programmed cell death of Drosophila central nervous system midline cells. Proc. Natl. Acad. Sci. USA 94, 5131-5136. 


\section{STAR $\star$ METHODS}

\section{KEY RESOURCES TABLE}

\begin{tabular}{|c|c|c|}
\hline REAGENT or RESOURCE & SOURCE & IDENTIFIER \\
\hline \multicolumn{3}{|l|}{ Chemicals, Peptides, and Recombinant Proteins } \\
\hline Rabbit anti-GFP & Molecular Probes & Cat\#: A-21312; RRID: AB_221478 \\
\hline Mouse anti-BRP & DSHB & Cat\#: NC82; RRID: AB_2314866 \\
\hline Alexa Fluor 488 goat anti-rabbit & Thermo Fisher Scientific & Cat\#: A-11008; RRID: AB_2532697 \\
\hline Cy5 goat anti-mouse & Thermo Fisher Scientific & Cat\#: A-10524; RRID: AB_2534033 \\
\hline $16 \%$ Paraformaldehyde aqueous solution & EMS & Cat\#: 15710 \\
\hline Normal goat serum & Life Tech & Cat\#: 16210-064; RRID: AB_2336990 \\
\hline \multicolumn{3}{|l|}{ Experimental Models: Organisms/Strains } \\
\hline D. melanogaster: $w^{1118}$ & Ryder et al., 2004 & BDSC: 3605 FlyBase: FBst0003605 \\
\hline D. melanogaster: Canton-S & Amita Sehgal & BDSC: 64349 FlyBase: FBst0064349 \\
\hline D. melanogaster: $C S x$-iso31 & This manuscript & N/A \\
\hline D. melanogaster: $w^{*} ; P\{$ nan-GAL4.K\}2 & $\begin{array}{l}\text { Bloomington Drosophila } \\
\text { Stock Center }\end{array}$ & BDSC: 24903 FlyBase: FBst0024903 \\
\hline D. melanogaster: $P\{U A S-h i d . Z\} 2 / C y O$ & $\begin{array}{l}\text { Bloomington Drosophila } \\
\text { Stock Center }\end{array}$ & BDSC: 65403 FlyBase: FBst0065403 \\
\hline D. melanogaster: $w^{*} ;$ P $\{$ UAS-TrpA1(B).K\}attP16 & $\begin{array}{l}\text { Bloomington Drosophila } \\
\text { Stock Center }\end{array}$ & BDSC: 26263 FlyBase: FBst0026263 \\
\hline D. melanogaster: $D A T^{f m n}$ & Kume et al., 2005 & FlyBase: FBal0197506 \\
\hline D. melanogaster: $n A C h R \alpha 4^{r y e}$ & Shi et al., 2014 & FlyBase: FBal0295291 \\
\hline D. melanogaster: $\mathrm{sss}^{P 1}$ (also known as qvi $\mathrm{FY}^{\mathrm{Y} 04063}$ ) & Koh et al., 2008 & BDSC: 16588 FlyBase: FBal0161515 \\
\hline D. melanogaster: tara ${ }^{5132}$ & Afonso et al., 2015 & FlyBase: FBal0305281 \\
\hline D. melanogaster: P\{Gad1-Gal4.3.098\}2/Cyo & Hamasaka et al., 2005 & BDSC: 51630 FlyBase: FBst0051630 \\
\hline D. melanogaster: $w^{1118} ;$ UAS-Gad1 shRNA & This manuscript & N/A \\
\hline D. melanogaster: $T b h^{n m 18}$ & Monastirioti et al., 1996 & FlyBase: FBal0061578 \\
\hline D. melanogaster: $p e r^{01}$ & Konopka and Benzer, 1971 & BDSC: 80928 FlyBase: FBst0080928 \\
\hline \multicolumn{3}{|l|}{ Oligonucleotides } \\
\hline $\begin{array}{l}\text { Gad1 shRNA: GAATTCTTAATCCACAGCCTTTAATGTGA } \\
\text { TTGTTGATGTCGCGTAAGCTAAGTTAATATACCATATCT } \\
\text { AGCTTACGCGACATCAACAATCGTACCTAAAGTGCCTA } \\
\text { ACATCATTATITCAGCCTTGAGAGTTGGGTATAAACTG } \\
\text { TCCGAGAGGTAGTTATATTCAAGCATACCTCTCGGACAG } \\
\text { TTATACCCAGCGAAATCTGGCGAGCTCGAG }\end{array}$ & This manuscript & $\mathrm{N} / \mathrm{A}$ \\
\hline \multicolumn{3}{|l|}{ Recombinant DNA } \\
\hline UAS-Gad1 shRNA & This manuscript & $\mathrm{N} / \mathrm{A}$ \\
\hline \multicolumn{3}{|l|}{ Software and Algorithms } \\
\hline MATLAB & MathWorks & $\begin{array}{l}\text { https://www.mathworks.com/ } \\
\text { products/matlab.html }\end{array}$ \\
\hline SleepLab & Joiner, 2016 & Gift from Dr. William Joiner, UCSD \\
\hline DAM FileScan & Trikinetics & https://trikinetics.com/ \\
\hline MATLAB GUI for generation of vibration stimuli & This manuscript & \\
\hline Fiji & Fiji & https://fiji.sc \\
\hline SAS & SAS Institute & https://www.sas.com/geohome \\
\hline Excel & Microsoft & $\begin{array}{l}\text { https://www,microsoft.com/en-us/ } \\
\text { microsoft-365/excel }\end{array}$ \\
\hline iMovie & Apple & $\begin{array}{l}\text { https://apps.apple.com/us/app/ } \\
\text { imovie/id377298193 }\end{array}$ \\
\hline
\end{tabular}

(Continued on next page) 


\begin{tabular}{lll}
\hline Continued & & \\
\hline REAGENT or RESOURCE & SOURCE & IDENTIFIER \\
\hline Prism 8 & GraphPad & scientific-software/prism/ \\
\hline Other & & DAM2 \\
\hline $\begin{array}{l}\text { Drosophila Activity Monitoring System } \\
\text { Single-beam Monitor }\end{array}$ & Trikinetics & MB5 \\
$\begin{array}{l}\text { Drosophila Activity Monitoring System } \\
\text { Multi-beam Monitor }\end{array}$ & Trikinetics & LC4 \\
\hline LC4 Light Controller & Trikinetics & Cat\#: 02-215-450 \\
Multi-tube vortexer & Fisher Scientific & SKU\#: PLPW15D \\
15" Car Subwoofer DVC 4-Ohm & Pyle Audio & SEN-12786 \\
Triple axis accelerometer breakout ADXL337 & Sparkfun & Cat\#: 15000-00 \\
\hline 3mm Vannas Spring Scissors & Fine Science Tool & Model\#: DR-36VL \\
Percival Incubator & Percival Scientific & Model\#: VRI20PD \\
VWR Incubator & VWR International & Model\#: USB-6001 \\
\hline Multifunction I/O Device (NI DAQ) & National Instruments & Model\#: TCS SP8 \\
\hline Leica SP8 Confocal Microscope & Leica Microsystems & \\
\hline
\end{tabular}

\section{RESOURCE AVAILABILITY}

\section{Lead Contact}

Further information and requests for resources and reagents should be directed to and will be fulfilled by the Lead Contact, Kyunghee Koh (kyunghee.koh@jefferson.edu).

\section{Material Availability}

All unique reagents generated in this study are available from the Lead Contact upon request without restriction.

\section{Data and Code Availability}

All data supporting the findings of this study are included within the article and Supplementary Information files. Data and the MAT$\mathrm{LAB}$ code for generating vibration stimuli are available from the Lead Contact upon request.

\section{EXPERIMENTAL MODEL AND SUBJECT DETAILS}

\section{Fly Stocks}

Flies were raised on standard food containing molasses, cornmeal, and yeast at $25^{\circ} \mathrm{C}$ under a $12-\mathrm{h}: 12-\mathrm{h}$ LD cycle. Adult flies, 3- to 5 day-old at the start of experiments, were used. Some experiments included males and females, whereas others included only females, as indicated in figure legends. iso31 ( $w^{1118}$ ), Canton-S (CS), and per ${ }^{01}$ (Konopka and Benzer, 1971) lines were obtained from Amita Sehgal, and CSx-iso31 was generated by replacing the X chromosome of iso31 with the X chromosome of CS. Fly lines carrying nan-Gal4 (\#24903) (Kim et al., 2003), UAS-hid (\#65403) (Zhou et al., 1997), UAS-dTrpA1 (\#26263) (Hamada et al., 2008), and Gad1-Gal4 (\#51630) (Hamasaka et al., 2005) were obtained from the Bloomington Drosophila Stock Center. DAT ${ }^{f m n}$ mutants were obtained from Kazuhiko Kume (Kume et al., 2005), $n A C h R \alpha 4^{\text {rye }}$ from Amita Sehgal (Shi et al., 2014), and Tbh ${ }^{\text {nm18 }}$ from Maria Monastirioti (Monastirioti et al., 1996). sss ${ }^{P 1}$ (Koh et al., 2008) and tara ${ }^{s 132}$ (Afonso et al., 2015) were described previously. Transgenic flies carrying UAS-Gad1 shRNA were generated in this study as described below. Fly lines were outcrossed to iso31 for at least five generations, except for CS and CSx-iso31.

\section{METHOD DETAILS}

\section{Sleep Analysis}

For sleep analysis, 3- to 5-day-old flies were entrained to either 12-h: 12-h LD or constant light (LL) for at least 3 days, and baseline sleep was measured $\sim 2$ days after being loaded into tubes. For DD experiments, flies were entrained to LD until loading, and baseline sleep was measured 1-2 days after being switched to DD. Approximately 16 males and 16 females were housed together until they were individually loaded into glass tubes containing $5 \%$ sucrose and $2 \%$ agar. Experiments were performed at $25^{\circ} \mathrm{C}$ except for thermogenetic experiments, in which flies were raised at $22^{\circ} \mathrm{C}$, monitored for 1 day at $22^{\circ} \mathrm{C}$ to determine baseline sleep levels, and 1 day at $29^{\circ} \mathrm{C}$ to activate the $\mathrm{dTrpA} 1$ channel. Activity data (beam breaks) were collected in 1-min bins using Drosophila Activity Monitoring 
(DAM) System (Trikinetics, Waltham, MA) to measure sleep defined as a period of inactivity lasting at least 5 min (Huber et al., 2004). Single-beam monitors were used except where the use of multi-beam monitors was specifically noted. For multi-beam data, the "counts" setting was used to detect the combined local, intra-beam movements and inter-beam movements, whereas the "moves" setting was used to detect inter-beam movements only. Intra-beam movements were calculated by subtracting moves from counts. Sleep parameters were analyzed using a custom MATLAB-based software SleepLab (Joiner, 2016).

\section{Generation of Vibration Stimuli}

Activity monitors, monitor tubes, and recording arenas containing flies were placed on a shelf $\sim 40 \mathrm{~cm}$ above an analog multi-tube vortexer (Fisher Scientific, Pittsburgh, PA) or secured on the speaker system platform using screws (Figures S1A and S6A). The vortexer and speaker system were placed in light- and temperature-controlled incubators (VWR International, Radnor, PA and DR-36VL, Percival Scientific, Perry, IA, respectively). For the vortexer experiments, the intensity was set to 3 , and the duration and timing of the mechanical stimulation was controlled via LC4 Light Controller (Trikinetics, Waltham, MA). For the speaker system experiments, a custom MATLAB GUI was used to generate audio signals of arbitrary frequency and amplitude. The DAM activity monitors were securely fastened to an acrylic platform that was glued to the cone of a 15-in marine subwoofer (PLPW15D, Pyle Audio, Brooklyn, NY). A PC delivered audio signals to the amplifier that powered the subwoofer, driving mechanical oscillations. The MATLAB GUI also collected acceleration data from a triple axis accelerometer breakout (ADXL337, Sparkfun Electronics, Niwot, CO) mounted on a platform via a data acquisition device (NI DAQ, National Instruments, Austin, TX).

\section{Video Recording and Quantification}

For Video S1, flies were loaded into $7 \mathrm{~mm} \times 16 \mathrm{~mm} \times 4 \mathrm{~mm}$ wells containing $5 \%$ sucrose and $2 \%$ agar. Videos were recorded with a digital camera (DCR-SX63, Sony, Tokyo, Japan) and edited using iMovie (Apple, Cupertino, CA). For quantitative video analysis of behavior, individual flies were loaded into standard DAM monitor tubes (65 mm x $5 \mathrm{~mm}$ glass tubes) containing $5 \%$ sucrose and $2 \%$ agar. Behavior was manually scored for the first hour of vibration starting at ZT 1 and the corresponding hour on the previous baseline day. Behavior was categorized as sleep ( $>5 \mathrm{~min}$ of inactivity), rest ( $<5 \mathrm{~min}$ and $>10 \mathrm{~s}$ of inactivity), locomotion, grooming, and eating. Inactivity lasting less than $10 \mathrm{~s}$ was categorized as the same behavior as the flanking period. Data from two experiments scored independently by two individuals showed the same pattern of results, and pooled data are shown.

\section{Analysis of Sensory Responsiveness}

Flies were exposed to extremely bright light ( 15,000 lux) of varying durations ( $1 \mathrm{~s}, 15 \mathrm{~s}$ and $1 \mathrm{~min})$ after being entrained under constant moderately bright light ( $\sim 500$ lux). Bright light stimuli were applied every 30 min during alternating periods of vibration (1 h) and no vibration (1 or $2 \mathrm{~h}$ ) using LC4 Light Controller (Trikinetics, Waltham, MA). The train of light stimulus started 25 min after the onset of the first vibration period. Sensory responsiveness was measured as the percentage of flies that started moving within 2 min of the bright light stimulus out of those that were asleep at the time of the stimulus presentation. Similar calculations were performed on data 10 min prior to light stimuli to measure spontaneous awakening. A series of 1 min dark pulses were applied every hour during alternating periods of $1 \mathrm{~h}$ vibration and $2 \mathrm{~h}$ silence, starting $45 \mathrm{~min}$ after the onset of the first vibration. Excel (Microsoft, Redmond, WA) was used to determine the percentage of sleeping flies that awakened within 2 min of bright light or dark pulses.

\section{Immunohistochemistry}

For whole mount immunostaining, female brains were fixed in $4 \%$ paraformaldehyde (PFA) for 60 min at RT. After three washes in PBT (0.3\% Triton-X in PBS), dissected brains were blocked in $1 \%$ normal goat serum in PBT for $1 \mathrm{~h}$ at RT and incubated with primary antibody at $4^{\circ} \mathrm{C}$ overnight. After three washes in PBT, they were incubated with secondary antibodies at $4^{\circ} \mathrm{C}$ overnight. Rabbit anti-GFP (Molecular Probes, Eugene, OR, Cat\# A-21312; RRID:AB_221478) was used at 1:1500, mouse anti-BRP (NC82, DSHB, lowa City, IA, Cat\# nc82; RRID: AB_2314866) at 1:200, Alexa Fluor 488 goat anti-rabbit (Thermo Fisher Scientific, Waltham, MA, Cat\# A11008; RRID:AB_2532697) at 1:1000, and Cy5 goat anti-mouse (Thermo Fisher Scientific, Waltham, MA, Cat\# A10524; RRID:AB_2534033) at 1:1000. A Leica SP8 confocal microscope (Leica Microsystems, Wetzlar, Germany) was used for imaging, and Fiji (https://fiji.sc) was used for image processing.

\section{Antennae Ablation}

All three antennal segments of 1- to 2-day-old flies were physically removed using $3 \mathrm{~mm}$ Vannas Spring Scissors (Fine Science Tools, Foster City, CA). After 3 days of recovery, flies were loaded into monitor tubes for sleep analysis.

\section{Transgenic Fly Lines}

To generate the UAS-Gad1 shRNA construct, 215bp containing two 21mers (GAT TGT TGA TGT CGC GTA AGC and GGG TAT AAA CTG TCC GAG AGG) in the coding region of Gad1 was designed as described (Chen et al., 2007) and synthesized by GeneArt (Regensburg, Germany). The synthetic DNA was inserted into the pUAST vector, and transgenic lines carrying the construct were generated by standard germline transformation in the iso31 background (Rainbow Transgenics, Camarillo, CA). 


\section{QUANTIFICATION AND STATISTICAL ANALYSIS}

Statistical tests were performed using GraphPad Prism 8 (GraphPad, San Diego, CA), except for two-way repeated-measures ANOVAs, as noted below. Paired Student's t tests with Bonferroni corrections were used to determine whether vibration induced significant changes in sleep and activity. For comparison of pairs of groups such as antennae ablated versus intact flies, unpaired Student's $t$ tests were performed, and for comparison of 3 or more groups, ANOVAs were performed. If the groups had unequal variances, Welch t tests for unequal variances or the Brown-Forsythe and Welsh version of ANOVAs were used. Following ANOVAs, Dunnett's or Sidak's posthoc tests were performed depending on the type and number of posthoc tests. Wilcoxon matched-pairs signed-rank tests with Bonferroni corrections were performed to analyze sleep bout duration data. For the analysis of arousability by light and dark pulses, $\chi$-square tests were performed, followed by Bonferroni correction for multiple tests. To compare continuous versus intermittent vibration blocks in Figure 5, we performed two-way repeated-measures ANOVAs using SAS v9.4 (SAS Institute, Cary, NC), with the training block as a within-subject factor and the order of continuous and intermittent vibration blocks as the between-subject factor. All interactions between the main factors were significant, and selected posthoc comparisons were corrected for multiple comparisons using the Bonferroni method. Details of statistical tests, including $p$ values and $n$, can be found in figure legends. All experiments were performed at least twice using flies from independent crosses. 